\title{
Cosmic acceleration with a negative cosmological constant in higher dimensions
}

\author{
Kei-ichi Maeda ${ }^{a}$ and Nobuyoshi Ohta ${ }^{b}$ \\ ${ }^{a}$ Department of Physics, Waseda University, \\ Shinjuku, Tokyo 169-8555, Japan \\ ${ }^{b}$ Department of Physics, Kinki University, \\ Higashi-Osaka, Osaka 577-8502, Japan \\ E-mail: maeda@waseda.ac.jp, ohtan@phys.kindai.ac.jp
}

ABStRaCT: We study gravitational theories with a cosmological constant and the GaussBonnet curvature squared term and analyze the possibility of de Sitter expanding spacetime with a constant internal space. We find that there are two branches of the de Sitter solutions: both the curvature of the internal space and the cosmological constant are (1) positive and (2) negative. From the stability analysis, we show that the de Sitter solution of the case (1) is unstable, while that in the case (2) is stable. Namely de Sitter solution in the present system is stable if the cosmological constant is negative. We extend our analysis to the gravitational theories with higher-order Lovelock curvature terms. Although the existence and the stability of the de Sitter solutions are very complicated and highly depend on the coupling constants, there exist stable de Sitter solutions similar to the case (2). We also find de Sitter solutions with Hubble scale much smaller than the scale of a cosmological constant, which may explain a discrepancy between an inflation energy scale and the Planck scale.

KeYwords: Cosmology of Theories beyond the SM, Supergravity Models, Superstrings and Heterotic Strings, Classical Theories of Gravity

ARXIV EPRINT: 1404.0561 


\section{Contents}

1 Introduction 1

2 Einstein-Gauss-Bonnet system with a cosmological constant 4

2.1 Field equations 4

2.2 Solutions of accelerating universe 5

$\begin{array}{lll}2.3 & \text { Stability of accelerating universe } & 7\end{array}$

3 Lovelock gravity $\quad 10$

$\begin{array}{ll}3.1 \text { Basic equations } & 10\end{array}$

$\begin{array}{ll}3.2 & \text { Perturbation equations } \\ 3.3 & \text { Mintowski }\end{array}$

$\begin{array}{lll}3.3 & \text { Minkowski spacetime } & 13\end{array}$

$\begin{array}{lll}3.4 & \text { Stability of near-Minkowski spacetime } & 14\end{array}$

4 De Sitter spacetimes with higher-order Lovelock terms and their stability 17

4.1 The effect of the quartic Lovelock term with $\alpha_{4}\left(\alpha_{3}=0\right) \quad 18$

4.2 The effect of the cubic Lovelock term with $\alpha_{3}\left(\alpha_{4}=0\right) \quad 22$

4.3 The effect of generic Lovelock terms $\left(\alpha_{3}, \alpha_{4} \neq 0\right) \quad 24$

5 Concluding remarks $\quad 26$

\section{Introduction}

It is now commonly believed that there is an inflationary epoch of the early stage of the evolution of our universe $[1-6] .^{1}$ This has been confirmed by the observation of the density fluctuation of the universe [8-14]. There is also a strong evidence that the present universe exhibits accelerating expansion. These facts prompt us to build cosmological models with accelerating phases. One may achieve this goal by introducing inflation with suitable potential. However it is more desirable if we can derive such models from the first principle or fundamental theory of particle physics without artificial assumptions. The most promising candidate of such a fundamental theory is the ten-dimensional superstring or eleven-dimensional $\mathrm{M}$ theory. However, it has been well known that an accelerating universe is difficult to realize for such theories, because there exists the so-called "no-go theorem" $[15,16]$, which forbids accelerated expanding spacetime solutions if an internal space is a time-independent nonsingular compact manifold without boundary.

Breaking some of the assumptions in the theorem, we can look for a natural inflationary scenario. One possibility is the brane inflation models $[17-20],{ }^{2}$ in which we assume

\footnotetext{
${ }^{1}$ See also [7] for a compact review.

${ }^{2}$ See also the following review article [21].
} 
test branes and do not take into account the back reactions. Another is the S-brane solutions [22, 23], in which temporal acceleration is possible but unfortunately big enough e-folding and/or long enough expansion was not obtained [24-27].

This suggests that the low-energy effective theory which is given by supergravity should have some modification of either the gravity side or matter side of the Einstein equation. A simple extension would be to introduce the cosmological constant, which must be extremely tiny to account for the current observation. From the supergravity point of view, this is not desirable because it is not natural to introduce such a tiny cosmological constant. Fortunately it is known that there are higher-order modifications to the low-energy gravitational action in superstrings. The leading corrections are given by the Gauss-Bonnet (GB) terms in heterotic string [28]-[31]. The effects of such terms have been studied in several papers, and interesting results are obtained that the inflationary universe is possible [32, 33], but some refinement was necessary to achieve enough inflation. Constraints on such models are also discussed in [34], where it was shown for flux compactification it is not possible to obtain de Sitter solutions. Earlier references on related subjects include [35]-[65].

In these works, no cosmological constant was considered. Recently it has been argued that when the curvature of the extra dimensional space is negative and there is a cosmological constant, one obtains solutions in which both the volume of the extra dimension and expansion rate of the four-dimensional spacetime tends to a constant [66]. Stability of the obtained solutions is also examined. Naively we expect that there is no cosmological constant in the effective low-energy theories of superstrings, but its existence is not excluded. For example, it is known that type IIA theories have a 10 -form whose expectation value may give rise to such a cosmological constant [67]. Other possible sources include generation of such a term at one-loop in non-supersymmetric heterotic string [68]. There are also various forms in superstrings which could produce similar terms. So this is an interesting possibility and the search for cosmological solutions in the theory deserves further study.

When these forms get expectation values in superstring theories, they typically produce negative cosmological constant. This is also compatible with supersymmetry. As we discuss below, it is precisely when we have the negative cosmological constant that we find stable (de Sitter spacetime) $\times$ (maximally symmetric space of constant size). So our following solutions would be naturally realized in superstring theories. However, we should note that though the theories we consider are well motivated by heterotic string, there are some differences like neglecting dilaton field for simplicity. Also in any higher-dimensional theories including superstrings, it is always an important issue how to stabilize the moduli after compactification. We do not address this difficult question in this paper though we examine the stability of the obtained solutions against small perturbations in the overall sizes of four-dimensional spacetime and extra dimensions. Thus our stability does not guarantee that the solutions are stable in all directions, but if they are unstable in our analysis, they do not give interesting solutions.

In this paper we study whether it is possible to obtain solutions with de Sitter expansion of the four-dimensional spacetime and static internal space within the theories with such higher-order terms and a cosmological constant. It was stated in [66] that for a wide range of parameters (cosmological constant, Einstein and GB term coefficients) it is verified numerically that there are solutions of this type, but the details are not clear including 
the question of for what range of parameters this type of solutions are possible. We intend to extend this work to include a comprehensive scan of the parameter space as well as higher order Lovelock terms: we exhaust all possible such solutions for arbitrary signs of cosmological constant and signatures of the (constant) curvatures of the internal spaces. We do this first for theories with GB term, but extend the analysis to the effects of further higher-order Lovelock gravity. We also examine the stability of the obtained solutions. What is most interesting is that we find that such solutions exist even for negative cosmological constant provided that the curvature of the internal space is negative. Moreover we can also have such solutions for positive cosmological constant but they are unstable in general, and those solutions for negative cosmological constant give stable solutions. We also find that this tendency of the existence of the solutions and stability persist in the presence of the higher order terms. Note that these solutions are quite different from those in the Einstein theory with a cosmological constant, where such solutions exist only for positive cosmological constant and they are unstable.

We should note that there are similar claims that modified gravity with a negative cosmological constant can have a positive effective cosmological constant [69] based on [70]. In the latter paper [70], in order to avoid eternal acceleration, a negative cosmological constant is introduced together with quintessence scalar field which has positive potential and gives positive contribution to the cosmological constant. This scalar degrees of freedom is interpreted as arising from the $f(R)$ gravity, which produces such scalar with exponential "cosmological term" in [69]. In this view, the negative cosmological constant is cancelled by the scalar potential even though it appears that one considers modified gravity. Our mechanism is different from this in that we do not introduce a positive potential (or terms which can be rewritten as a potential). In another work [71], Wheeler-De Wit equation was studied semiclassically with a similar result.

This paper is organized as follows: in section 2, we begin with the Einstein-GaussBonnet theory with a cosmological constant. First we present our basic equations in this theory. For comparison, we summarize solutions of accelerating universe of the form (de Sitter spacetime $) \times($ maximally symmetric space $)$ in the Einstein gravity with a cosmological constant. Then in the present theory with GB term, we find solutions and study their stability. We then proceed to the study of the effects of higher Lovelock gravity in section 3. We give the basic equations for the above spacetime in subsection 3.1, the equations for perturbation in subsection 3.2, and give solutions with the de Sitter spacetime being Minkowski in subsection 3.3. We then examine the stability of the solutions, and determine the region of parameters $\alpha_{3}$ and $\alpha_{4}$ for the stable Minkowski solutions in subsection 3.4. In section 4, we discuss the solutions of the form (de Sitter spacetime) $\times$ (maximally symmetric space of constant size) including the effects of higher-order Lovelock gravity. Section 5 is devoted to our conclusion. 


\section{Einstein-Gauss-Bonnet system with a cosmological constant}

\subsection{Field equations}

We consider the following low-energy effective action for the heterotic string with a cosmological constant $\Lambda$ :

$$
S=\frac{1}{2 \kappa_{D}^{2}} \int d^{D} x \sqrt{-g}\left[R-2 \Lambda+\alpha_{2} R_{\mathrm{GB}}^{2}\right],
$$

where $\kappa_{D}^{2}$ is a $D$-dimensional gravitational constant, $\alpha_{2}=\alpha^{\prime} / 8$ is a numerical coefficient given in terms of the Regge slope parameter, and $R_{\mathrm{GB}}^{2}=R_{\mu \nu \rho \sigma} R^{\mu \nu \rho \sigma}-4 R_{\mu \nu} R^{\mu \nu}+R^{2}$ is the GB correction. Here the two-form and gauge fields (and their higher order terms) are dropped because setting them to zero is consistent with field equations. We also neglect dilaton for simplicity.

Let us consider the metric in $D$-dimensional space,

$$
d s_{D}^{2}=-e^{2 u_{0}(t)} d t^{2}+e^{2 u_{1}(t)} d s_{p}^{2}+e^{2 u_{2}(t)} d s_{q}^{2},
$$

where $D=1+p+q$. The external $p$-dimensional and internal $q$-dimensional spaces $\left(d s_{p}^{2}\right.$ and $d s_{q}^{2}$ ) are chosen to be maximally symmetric, with the signature of the curvature given by $\sigma_{p}$ and $\sigma_{q}$, respectively. Though we are mainly concerned with flat external space $\left(\sigma_{p}=0\right)$ in this paper, it may be useful to give field equations for more general case.

The Ricci scalar and the GB correction term are given by

$$
\begin{aligned}
R= & e^{-2 u_{0}}\left[p_{1} A_{p}+q_{1} A_{q}-2\left(p_{1} \dot{u}_{1}^{2}+p q \dot{u}_{1} \dot{u}_{2}+q_{1} \dot{u}_{2}^{2}\right)\right] \\
R_{\mathrm{GB}}^{2}= & e^{-4 u_{0}}\left\{p_{3} A_{p}^{2}+2 p_{1} q_{1} A_{p} A_{q}+q_{3} A_{q}^{2}+4 \dot{u}_{1} \dot{u}_{2}\left(p_{2} q A_{p}+p q_{2} A_{q}\right)+4 p_{1} q_{1} \dot{u}_{1}^{2} \dot{u}_{2}^{2}\right. \\
& \left.+4 p X\left[(p-1)_{2} A_{p}+q_{1} A_{q}+2(p-1) q \dot{u}_{1} \dot{u}_{2}\right]+4 q Y\left[p_{1} A_{p}+(q-1)_{2} A_{q}+2 p(q-1) \dot{u}_{1} \dot{u}_{2}\right]\right\},
\end{aligned}
$$

where

$$
\begin{aligned}
A_{p} & :=\dot{u}_{1}^{2}+\sigma_{p} e^{2\left(u_{0}-u_{1}\right)}, & A_{q} & :=\dot{u}_{2}^{2}+\sigma_{q} e^{2\left(u_{0}-u_{2}\right)}, \\
X & :=\ddot{u}_{1}-\dot{u}_{0} \dot{u}_{1}+\dot{u}_{1}^{2}, & Y & :=\ddot{u}_{2}-\dot{u}_{0} \dot{u}_{2}+\dot{u}_{2}^{2} .
\end{aligned}
$$

We have also used the following abbreviation:

$$
(k-\ell)_{m}:=(k-\ell)(k-\ell-1)(k-\ell-2) \cdots(k-m),
$$

where $k, \ell, m$ are integer numbers with $k>\ell, k>m$ and $\ell<m$.

Now the field equations are [32]

$$
\begin{aligned}
F & :=F_{1}+F_{2}=0 \\
F^{(p)} & :=f_{1}^{(p)}+f_{2}^{(p)}+X\left(g_{1}^{(p)}+g_{2}^{(p)}\right)+Y\left(h_{1}^{(p)}+h_{2}^{(p)}\right)=0, \\
F^{(q)} & :=f_{1}^{(q)}+f_{2}^{(q)}+Y\left(g_{1}^{(q)}+g_{2}^{(q)}\right)+X\left(h_{1}^{(q)}+h_{2}^{(q)}\right)=0,
\end{aligned}
$$

where

$$
\begin{aligned}
F_{1} & =p_{1} A_{p}+q_{1} A_{q}+2 p q \dot{u}_{1} \dot{u}_{2}-2 \Lambda e^{2 u_{0}}, \\
f_{1}^{(p)} & =(p-1)_{2} A_{p}+q_{1} A_{q}+2(p-1) q \dot{u}_{1} \dot{u}_{2}-2 \Lambda e^{2 u_{0}}, \quad f_{1}^{(q)}=p_{1} A_{p}+(q-1)_{2} A_{q}+2 p(q-1) \dot{u}_{1} \dot{u}_{2}-2 \Lambda e^{2 u_{0}},
\end{aligned}
$$




$$
g_{1}^{(p)}=2(p-1), \quad g_{1}^{(q)}=2(q-1), \quad h_{1}^{(p)}=2 q, \quad h_{1}^{(q)}=2 p,
$$

and

$$
\begin{aligned}
F_{2} & =\alpha_{2} e^{-2 u_{0}}\left\{p_{3} A_{p}^{2}+2 p_{1} q_{1} A_{p} A_{q}+q_{3} A_{q}^{2}+4\left(p_{2} q A_{p}+p q_{2} A_{q}+p_{1} q_{1} \dot{u}_{1} \dot{u}_{2}\right) \dot{u}_{1} \dot{u}_{2}\right\}, \\
f_{2}^{(p)} & =\alpha_{2} e^{-2 u_{0}}\left\{(p-1)_{4} A_{p}^{2}+2(p-1)_{2} q_{1} A_{p} A_{q}+q_{3} A_{q}^{2}+4\left[(p-1)_{3} q A_{p}+(p-1) q_{2} A_{q}+(p-1)_{2} q_{1} \dot{u}_{1} \dot{u}_{2}\right] \dot{u}_{1} \dot{u}_{2}\right\}, \\
f_{2}^{(q)} & =\alpha_{2} e^{-2 u_{0}}\left\{p_{3} A_{p}^{2}+2 p_{1}(q-1)_{2} A_{p} A_{q}+(q-1)_{4} A_{q}^{2}+4\left[p_{2}(q-1) A_{p}+p(q-1)_{3} A_{q}+p_{1}(q-1)_{2} \dot{u}_{1} \dot{u}_{2}\right] \dot{u}_{1} \dot{u}_{2}\right\}, \\
g_{2}^{(p)} & =4(p-1) \alpha_{2} e^{-2 u_{0}}\left[(p-2)_{3} A_{p}+q_{1} A_{q}+2(p-2) q \dot{u}_{1} \dot{u}_{2}\right], \\
g_{2}^{(q)} & =4(q-1) \alpha_{2} e^{-2 u_{0}}\left[p_{1} A_{p}+(q-2)_{3} A_{q}+2 p(q-2) \dot{u}_{1} \dot{u}_{2}\right], \\
h_{2}^{(p)} & =4 q \alpha_{2} e^{-2 u_{0}}\left[(p-1)_{2} A_{p}+(q-1)_{2} A_{q}+2(p-1)(q-1) \dot{u}_{1} \dot{u}_{2}\right], \\
h_{2}^{(q)} & =4 p \alpha_{2} e^{-2 u_{0}}\left[(p-1)_{2} A_{p}+(q-1)_{2} A_{q}+2(p-1)(q-1) \dot{u}_{1} \dot{u}_{2}\right] .
\end{aligned}
$$

The basic relations, eqs. (2.7)-(2.9), are not all independent as they satisfy

$$
\dot{F}+\left(p \dot{u}_{1}+q \dot{u}_{2}-2 \dot{u}_{0}\right) F=p \dot{u}_{1} F^{(p)}+q \dot{u}_{2} F^{(q)} .
$$

Here we normalize the variables by $\alpha_{2}$ such that $\tilde{A}_{q}=\alpha_{2} A_{q}, \tilde{\Lambda}=\alpha_{2} \Lambda$ and $\tilde{t}=t / \sqrt{\alpha_{2}}$. In what follows, we drop a tilde for brevity.

\subsection{Solutions of accelerating universe}

In this section, we solve the equations and provide an accelerating universe with a constant internal space. Thus we assume

$$
\dot{u}_{1}=H, \quad \dot{u}_{2}=0 .
$$

We choose the time coordinate as $u_{0}=0$ and take the Hubble parameter $H$ to be constant and the curvature of external space to be zero $\left(\sigma_{p}=0\right)$. The latter condition in $(2.13)$ means that $A_{q}$ is also constant. Then the basic equations turn to be algebraic:

$$
\begin{aligned}
-2 \Lambda+p_{1} H^{2}+q_{1} A_{q}+p_{3} H^{4}+2 p_{1} q_{1} H^{2} A_{q}+q_{3} A_{q}^{2} & =0, \\
-2 \Lambda+p(p+1) H^{2}+(q-1){ }_{2} A_{q}+(p+1)_{2} H^{4}+2 p(p+1)(q-1){ }_{2} H^{2} A_{q}+(q-1)_{4} A_{q}^{2} & =0 .
\end{aligned}
$$

Usually, for a given cosmological constant $\Lambda$, we obtain $H^{2}$ and $A_{q}$ by solving these coupled quadratic equations. There is a simpler way to find solutions in our case: we can solve the equations for $H^{2}$ and $\Lambda$ for given $A_{q}$, which are just a single quadratic (or linear) equation in $H^{2}$ and a linear equation in $\Lambda$ :

$$
\begin{aligned}
& 2 p_{2} H^{4}+p H^{2}\left[1-2(q-1)(p-q+1) A_{q}\right]-(q-1) A_{q}\left[1+2(q-2)_{3} A_{q}\right]=0, \\
& 2 \Lambda=p_{3} H^{4}+p_{1} H^{2}\left(1+2 q_{1} A_{q}\right)+q_{1} A_{q}\left[1+(q-2){ }_{3} A_{q}\right] .
\end{aligned}
$$

Before going into the discussions of the solutions in the present model, we summarize the results in the case without GB term. When we have only the Einstein-Hilbert term with a cosmological constant, after compactification, we find $[a(p+1)$-dimensional de 
Sitter spacetime $] \times[$ a constant internal space with a positive curvature], if a cosmological constant is positive. The solution is given by

$$
\begin{aligned}
H & =\sqrt{\frac{2 \Lambda}{p(p+q-1)}} \\
A_{q} & =\frac{2 \Lambda}{(q-1)(p+q-1)} .
\end{aligned}
$$

The stability analysis, which we will show the detail later, gives two eigenvalues of perturbations:

$$
\omega_{ \pm}=\frac{H}{2}\left[-(p+q-1) \pm \sqrt{(p+q-1)^{2}+8 p}\right],
$$

one of which is always positive, giving an instability of this solution. There is no stable de Sitter solution.

Now let us discuss our model with the GB term. When $q=1, H=0$ is a trivial solution. Since $\sigma_{q}=0$, it is locally a Minkowski spacetime. To have a real solution for $H$, $H^{2}$ has to be real and positive. For $q \geq 2$, the possibly positive solution is

$$
\begin{aligned}
H^{2}= & \frac{1}{4 p_{2}}\left\{-p\left[1-2(q-1)(p-q+1) A_{q}\right]\right. \\
& \left.+\left[p^{2}\left[1-2(q-1)(p-q+1) A_{q}\right]^{2}+8 p_{2}(q-1) A_{q}\left[1+2(q-2){ }_{3} A_{q}\right]\right]^{1 / 2}\right\} .
\end{aligned}
$$

It is now easy to see that the condition for the existence of the real positive solutions of $H^{2}$ is $A_{q}\left[1+2(q-2)_{3} A_{q}\right] \geq 0$, which gives either $A_{q} \geq 0$, or

$$
A_{q} \leq A_{q}^{(\mathrm{M})}:=-\frac{1}{2(q-2)_{3}},
$$

when $q \geq 4$. We call the former and latter cases the branch (1) and the branch (2), respectively. For $q=2$ or 3 , we have only the branch (1) with $A_{q} \geq 0$.

The cosmological constant is given by eq. (2.16). This is one parameter $\left(A_{q}\right)$ family of solutions.

Let us show some example in the case of $p=3, q=6$ in figures 1 and 2. Figure 1 shows the Hubble expansion parameter square $H^{2}$ in terms of $A_{6}$, which is the solution of eq. (2.19), while figure 2 gives $H^{2}$ and $A_{6}$ in terms of a cosmological constant given by (2.16).

The cosmological constant is always positive for the branch (1) solutions with $A_{q} \geq 0$. On the other hand, for the branch (2) solutions with $A_{q}<0$, we find

$$
\Lambda \leq \Lambda^{(\mathrm{M})}:=-\frac{q_{1}}{8(q-2)_{3}}
$$

which is always negative. Here the equality corresponds to the Minkowski spacetime $(H=$ 0 ) with negative $A_{q}^{(\mathrm{M})}$. It is remarkable that we have de Sitter solution even for a negative cosmological constant. We emphasize that this becomes possible due to the negative $A_{q}$ and the existence of the GB term. 


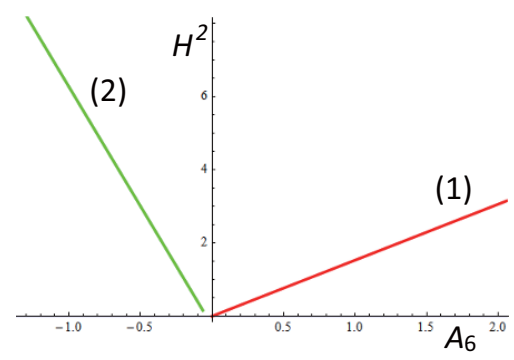

Figure 1. The Hubble parameter of de Sitter solution $H^{2}$ in terms of $A_{6}$ for $p=3, q=6$. There are two branches (1) and (2).

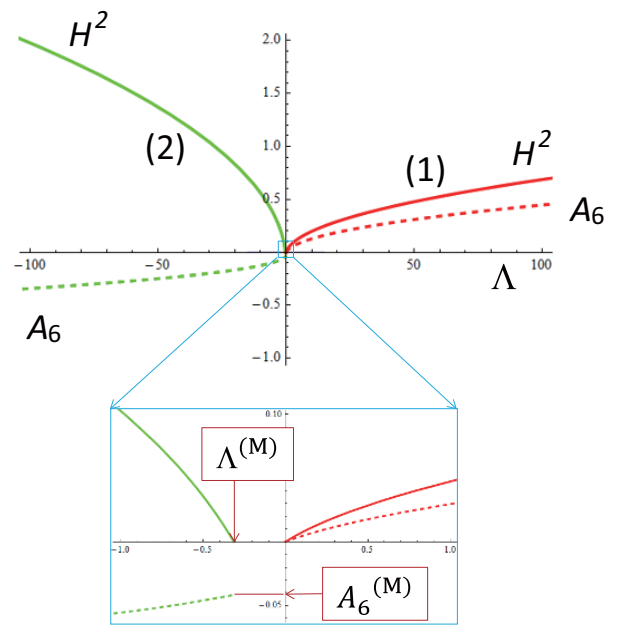

Figure 2. The Hubble parameter $H^{2}$ (sold curve) and the curvature of the internal space $A_{6}$ (dashed curve) in terms of $\Lambda$. The enlarged figure near the origin is shown at the bottom.

\subsection{Stability of accelerating universe}

Next we study the stability of the above solutions against small perturbations in the size of the spaces. Unless they are stable in these directions, they do not give interesting solutions. Choosing the time coordinate as $u_{0}=0$ and perturbing the variables around the background solution $\left(H, A_{q}\right)$ with $\Lambda$, given by eqs. (2.15) and (2.16), as

$$
\begin{aligned}
& u_{1}(t)=H t+\xi(t), \\
& u_{2}(t)=u_{2}^{(0)}+\eta(t),
\end{aligned}
$$

where $u_{2}^{(0)}$ is a constant and satisfies $A_{q}=\sigma_{q} e^{-2 u_{2}^{(0)}}$, we obtain the perturbation equations from our basic equations (2.7)-(2.9):

$$
\begin{aligned}
P \dot{\xi}+Q \dot{\eta}+R \eta & =0, \\
J \ddot{\xi}+K \ddot{\eta}+L \dot{\xi}+M \dot{\eta}+N \eta & =0, \\
S \ddot{\xi}+T \ddot{\eta}+U \dot{\xi}+V \dot{\eta}+W \eta & =0,
\end{aligned}
$$

where

$$
P:=p_{1} H X
$$




$$
\begin{aligned}
Q & :=p q H Y, \\
R & :=-p q H^{2} Y, \\
J & :=(p-1) X, \\
K & :=q Y, \\
L & :=p_{1} H X, \\
M: & =(p-1) q H Y, \\
N & :=-p q H^{2} Y, \\
S & :=p Y, \\
T & :=\frac{p H^{2}}{A_{q}} Y, \\
U: & =(p+1)_{0} H Y, \\
V & :=\frac{p^{2} H^{3}}{A_{q}} Y \\
W & :=-(q-1)_{2} A_{q} Z,
\end{aligned}
$$

with

$$
\begin{aligned}
& X:=1+2\left[(p-2)_{3} H^{2}+q_{1} A_{q}\right], \\
& Y:=1+2\left[(p-1)_{2} H^{2}+(q-1)_{2} A_{q}\right], \\
& Z:=1+2\left[p(p+1) H^{2}+(q-3)_{4} A_{q}\right] .
\end{aligned}
$$

Here we have used the equation for the background solution (2.15).

Eq. (2.24) is derived from eq. (2.23), which is guaranteed by the Bianchi identity (2.12). Hence the independent equations are eqs. (2.23) and (2.25). Eliminating $\dot{\xi}$ by use of eq. (2.23), we find the equation for $\eta$ as

$$
\ddot{\eta}+p H \dot{\eta}+C=0,
$$

where

$$
\begin{aligned}
C & :=\frac{P W-R U}{P T-S Q} \\
& =\frac{A_{q}\left[(p+1)_{0} q H^{2} Y^{2}-(p-1)(q-1)_{2} A_{q} X Z\right]}{p Y\left[(p-1) H^{2} X-q A_{q} Y\right]} .
\end{aligned}
$$

To analyze the stability, we set

$$
\eta=\eta_{0} e^{\omega t},
$$

to find a quadratic equation for the eigenvalue $\omega$ :

$$
\omega^{2}+p H \omega+C=0
$$

whose solutions are given by

$$
\omega=\omega_{ \pm}:=\frac{1}{2}\left(-p H \pm \sqrt{p^{2} H^{2}-4 C}\right)
$$




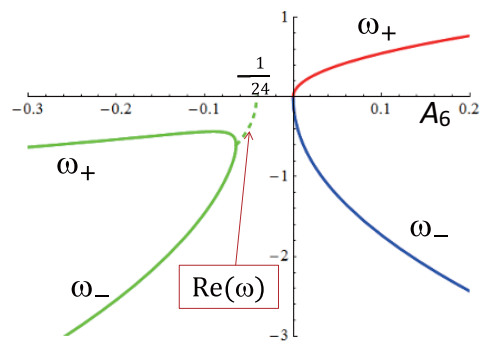

Figure 3. The eigenvalues $\omega_{ \pm}$and $\operatorname{Re}(\omega)$ in terms of $A_{6}$. The stable solution $\left(A_{6}<-1 / 24\right)$ has two real negative or a positive real part of two complex conjugate eigenvalues, which are shown by the green solid or dashed curves, respectively. The unstable solution $\left(A_{6}>0\right)$ has one real positive and one negative eigenvalues, which are shown by the red and blue solid curves, respectively.

If both eigenvalues $\omega_{ \pm}$are negative, i.e.,

$$
p^{2} H^{2}-4 C \geq 0 \text { and } C>0,
$$

or they are complex conjugates of each other with negative real part (guaranteed by $p H>$ $0)$, i.e.,

$$
p^{2} H^{2}-4 C<0
$$

the solution for the expanding universe $(H>0)$ is stable. Hence we conclude the expanding universe is stable if $C>0$.

Using the background solutions, we have studied their stability. For $q=1$, we have only Minkowski spacetime. No perturbation is possible. So we proceed to the case of $q \geq 2$.

First, we show one example of the eigenvalues $\omega$ for the case of $p=3$ and $q=6$ in figure 3.

For other dimensions, we also find similar results. The solution $H^{2}$ with positive $A_{q}$ is unstable because the perturbations have always one positive eigenvalue $\omega_{+}$. On the other hand, the solution $H^{2}$ with negative $A_{q}\left(\leq A_{q}^{(\mathrm{M})}\right)$ is stable.

Since the results in figure 3 is obtained by numerical calculation only for $p=3, q=6$, it is worth showing the results for general $p$ and $q$ in some simple limit. The Minkowski spacetime $(H=0)$ gives the boundary of a set of the solutions. Hence it may be important to analyze solutions near the Minkowski spacetime, which are given by

$$
H^{2} \approx \begin{cases}\frac{q-1}{p} A_{q} & \text { for } \operatorname{branch}(1)\left(A_{q} \geq 0\right) \\ -\frac{(q-1)_{3}}{p\left[(q-1)(p-q+1)+(q-2)_{3}\right]}\left(A_{q}-A_{q}^{(\mathrm{M})}\right) & \text { for } \operatorname{branch}(2)\left(A_{q} \leq A_{q}^{(\mathrm{M})}\right)\end{cases}
$$

assuming $H^{2} \ll 1$.

Using these solutions, we find

$$
C= \begin{cases}-2 p H^{2}+O\left(H^{4}\right) & \text { for } \operatorname{branch}(1), \\ \frac{(p-1)(q-1)(2 q-3)}{2 p q(q-2)^{2}}+O\left(H^{2}\right) & \text { for } \operatorname{branch}(2),\end{cases}
$$




\begin{tabular}{|c|c|c|c|c|}
\hline$q$ & branch & $A_{q}$ & $\Lambda$ & stability \\
\hline$q=1$ & - & No & No & - \\
\hline$q=2,3$ & $(1)$ & $A_{q} \geq 0$ & $\Lambda \geq 0$ & unstable \\
\hline$q \geq 4$ & $(1)$ & $A_{q} \geq 0$ & $\Lambda \geq 0$ & unstable \\
\cline { 2 - 5 } & $(2)$ & $A_{q} \leq A_{q}^{(\mathrm{M})}=-\frac{1}{2(q-2)_{3}}$ & $\Lambda \leq \Lambda^{(\mathrm{M})}=-\frac{q_{1}}{8(q-2)_{3}}$ & stable \\
\hline
\end{tabular}

Table 1. The range of $A_{q}$ where de Sitter solutions $(2.19)\left(H^{2}>0\right)$ exist.

which gives the eigenvalue as

$$
\omega=\omega_{ \pm}:= \begin{cases}\frac{1}{2}[-p \pm \sqrt{p(8+p)}] H+O\left(H^{2}\right) & \text { for } \operatorname{branch}(1) \\ -\frac{p H}{2} \pm \frac{i}{q-2} \sqrt{\frac{(p-1)(q-1)(2 q-3)}{2 p q}}+O\left(H^{2}\right) & \text { for } \operatorname{branch}(2)\end{cases}
$$

For the branch (1), the mode $\omega_{-}$is negative but the other mode $\omega_{+}$is positive. Hence the solution is unstable. On the other hand, for the branch (2), both modes $\omega_{ \pm}$have a negative real part for $H>0$. So the solution is stable. We conclude that the branch (2) solutions with negative $A_{q}$ are always stable, while the branch (1) solutions with positive $A_{q}$ are unstable in all dimensions we studied. As a result, we obtain the very interesting result that the solutions with a negative cosmological constant is always stable while those with a positive cosmological constant is unstable.

We summarize the existence conditions for de Sitter solutions in the present model and their stability in table 1.

\section{Lovelock gravity}

The preceding sections discussed the case only with $\alpha_{2}$, which is known as the next leading contribution in heterotic string theory. Here we consider the effects of further higher-order Lovelock gravity.

\subsection{Basic equations}

We consider the following action:

$$
S=\frac{1}{2 \kappa_{D}^{2}} \int d^{D} x \sqrt{-g} \sum_{n=0}^{n_{\max }} \alpha_{n} L_{n},
$$

where $n$-th order Lovelock terms $L_{n}$ are given by

$$
L_{n}:=\frac{1}{2^{n}} \delta_{j_{1} \cdots j_{2 n}}^{i_{1} \cdots i_{2 n}} R_{i_{1} i_{2}}^{j_{1} j_{2}} \cdots R_{i_{2 n-1} i_{2 n}}^{j_{2 n-1} j_{2 n}},
$$

$\alpha_{n}$ 's are their coupling constants with $\alpha_{1}=1$, and $L_{0}=1, L_{1}=R$ and $L_{2}=R_{\mathrm{GB}}^{2}$. We set $\alpha_{0}=-2 \Lambda$, where $\Lambda$ is a cosmological constant. Note that $n \leq n_{\max }:=[(D-1) / 2]$, beyond which no dynamical contributions by Lovelock terms exist. 
Assuming our spacetime is (de Sitter spacetime) $\times$ (a static maximally symmetric space), we find the field equations with $n$-th order Lovelock gravity terms [72]:

$$
\begin{aligned}
-2 \Lambda+\sum_{n=1}^{n_{\max }} \alpha_{n} \sum_{k=0}^{n}{ }_{n} C_{k} \frac{(p+1) !}{(p+1-2 n+2 k) !} \frac{(q-1) !}{(q-1-2 k) !} H^{2(n-k)} A_{q}^{k}=0, \\
-2 \Lambda+\sum_{n=1}^{n_{\max }} \alpha_{n} \sum_{k=0}^{n}{ }_{n} C_{k} \frac{p !}{(p-2 n+2 k) !} \frac{q !}{(q-2 k) !} H^{2(n-k)} A_{q}^{k}=0 .
\end{aligned}
$$

In eqs. (3.3) and (3.4), nontrivial terms exist for $n-[(p+1) / 2] \leq k \leq[(q-1) / 2]$ and $n-[p / 2] \leq k \leq[q / 2]$, respectively. Hence, the power exponents of $H^{2}$ and $A_{q}$ satisfy $(n-k) \leq[(p+1) / 2]$ and $k \leq[(q-1) / 2]$ in eq. (3.3) and $(n-k) \leq[p / 2]$ and $k \leq[q / 2]$ in eq. (3.4). As a result, eqs. (3.3) and (3.4) are quadratic equations for $H^{2}$ if $p \leq 4$ and those for $A_{q}$ if $q \leq 5$, respectively. Eliminating $\Lambda$ from eqs. (3.3) and (3.4), we find

$$
\begin{aligned}
H^{4} & {\left[\sum_{n=2}^{n_{\max }} \alpha_{n} \frac{n(n-1)}{2} \frac{p_{2}[2 D-n(p+1)](q-1) !}{(q-2 n+4) !} A_{q}^{n-2}\right] } \\
& +H^{2}\left[\sum_{n=1}^{n_{\max }} \alpha_{n} \frac{n p[D-n(p+1)](q-1) !}{(q-2 n+2) !} A_{q}^{n-1}\right]-\sum_{n=1}^{n_{\max }} \alpha_{n} \frac{n(q-1) !}{(q-2 n) !} A_{q}^{n}=0
\end{aligned}
$$

if $p \leq 4$, and

$$
\begin{aligned}
A_{q}^{2} & {\left[\sum_{n=2}^{n_{\max }} \alpha_{n} \frac{n(n-1)}{2} \frac{(q-1)_{3}(n q-2 D) p !}{(p-2 n+5) !} H^{2(n-2)}\right] } \\
& +A_{q}\left[\sum_{n=1}^{n_{\max }} \alpha_{n} \frac{n(q-1)(n q-D) p !}{(p-2 n+3) !} H^{2(n-1)}\right]+\sum_{n=1}^{n_{\max }} \alpha_{n} \frac{n p !}{(p-2 n+1) !} H^{2 n}=0(
\end{aligned}
$$

if $q \leq 5$. We can easily solve these quadratic equations. For the obtained solution of $H^{2}$ in terms of $A_{q}(p \leq 4)$, or that of $A_{q}$ in terms of $H^{2}(q \leq 5)$, the cosmological constant is explicitly given by one variable as

$$
2 \Lambda=\sum_{n=1}^{n_{\max }} \alpha_{n} \sum_{k=0}^{n}{ }_{n} C_{k} \frac{p !}{(p-2 n+2 k) !} \frac{q !}{(q-2 k) !} H^{2(n-k)} A_{q}^{k} .
$$

We then obtain one parameter family of analytic solutions: $H^{2}\left(A_{q}\right)$ and $\Lambda\left(A_{q}\right)$ for $p \leq 4$, or $A_{q}\left(H^{2}\right)$ and $\Lambda\left(H^{2}\right)$ for $q \leq 5$.

Note that the above ansatz of $p \leq 4$ or $q \leq 5$ is not so strong restriction. Superstring theory and M-theory predict $\mathrm{D}=10$ and 11 , respectively, for which dimensions we find either $p \leq 4$ or $q \leq 5$ because $p+q=D-1 \leq 10$. Hence, for such fundamental theories, we always find one parameter family of analytic solutions.

In what follows, we discuss the first case with $p \leq 4$ because it includes the realistic dimension $p=3$. We also consider only cubic and quartic Lovelock terms. It is the most general case for ten-dimensional superstring theory because $n_{\max }=4$ for $D=10$. Although we should include further higher-order Lovelock terms for the theories in dimension higher 
than ten such as M-theory, we may ignore those higher-order terms if the Lovelock terms originate from quantum corrections.

The quadratic equation (3.5) and the cosmological constant (3.7) are explicitly given as follows:

$$
\begin{aligned}
& p_{2} H^{4}\left[2 \alpha_{2}-3 \alpha_{3}(q-1)(p-2 q+1) A_{q}-12 \alpha_{4}(q-1)_{3}(p-q+1) A_{q}^{2}\right] \\
& \quad+p H^{2}\left[1-2 \alpha_{2}(q-1)(p-q+1) A_{q}-3 \alpha_{3}(q-1)_{3}(2 p-q+2) A_{q}^{2}-4 \alpha_{4}(q-1)_{5}(3 p-q+3) A_{q}^{3}\right] \\
& \quad-(q-1) A_{q}\left[1+2 \alpha_{2}(q-2)_{3} A_{q}+3 \alpha_{3}(q-2)_{5} A_{q}^{2}+4 \alpha_{4}(q-2)_{7} A_{q}^{3}\right]=0 . \\
& 2 \Lambda=p_{3} H^{4}\left[\alpha_{2}+3 \alpha_{3} q_{1} A_{q}+6 \alpha_{4} q_{3} A_{q}^{2}\right]+p_{1} H^{2}\left[1+2 \alpha_{2} q_{1} A_{q}+3 \alpha_{3} q_{3} A_{q}^{2}+4 \alpha_{4} q_{5} A_{q}^{3}\right] \\
& \quad+q_{1} A_{q}\left[1+\alpha_{2}(q-2)_{3} A_{q}+\alpha_{3}(q-2)_{5} A_{q}^{2}+\alpha_{4}(q-2)_{7} A_{q}^{3}\right] .
\end{aligned}
$$

When we include the GB term, the coefficient $\alpha_{2}$ must be positive in order to avoid a ghost. Hence we normalize the variables and coupling constants by $\alpha_{2}$ as

$$
\begin{aligned}
& \tilde{H}=\sqrt{\alpha_{2}} H, \quad \tilde{A}_{q}=\alpha_{2} A_{q}, \quad \tilde{\Lambda}=\alpha_{2} \Lambda, \\
& \tilde{\alpha}_{3}=\frac{\alpha_{3}}{\alpha_{2}^{2}}, \quad \tilde{\alpha}_{4}=\frac{\alpha_{4}}{\alpha_{2}^{3}} .
\end{aligned}
$$

In what follows, we drop a tilde for brevity.

\subsection{Perturbation equations}

In order to analyze stability, we perturb the basic equations. Here we consider general case with $n \leq 4$. We find two independent perturbation equations:

$$
\begin{aligned}
P \dot{\xi}+Q \dot{\eta}+R \eta & =0, \\
S \ddot{\xi}+T \ddot{\eta}+U \dot{\xi}+V \dot{\eta}+W \eta & =0,
\end{aligned}
$$

where the coefficients are defined by

$$
\begin{aligned}
P & :=p_{1} H X, \\
Q & :=p q H Y, \\
R & :=-p q H^{2} Y, \\
S & :=p Y, \\
T & :=\frac{p H^{2}}{A_{q}} Y, \\
U & :=(p+1)_{0} H Y, \\
V & :=\frac{p^{2} H^{3}}{A_{q}} Y, \\
W & :=-(q-1)_{2} A_{q} Z,
\end{aligned}
$$

with

$$
\begin{aligned}
X:= & 1+2\left((p-2)_{3} H^{2}+q_{1} A_{q}\right)+3 \alpha_{3}\left((p-2)_{5} H^{4}+2(p-2)_{3} q_{1} H^{2} A_{q}+q_{3} A_{q}^{2}\right) \\
& +4 \alpha_{4}\left((p-2)_{7} H^{6}+3(p-2)_{5} q_{1} H^{4} A_{q}+3(p-2)_{3} q_{3} H^{2} A_{q}^{2}+q_{5} A_{q}^{3}\right), \\
Y:= & 1+2\left((p-1)_{2} H^{2}+(q-1)_{2} A_{q}\right)+3 \alpha_{3}\left((p-1)_{4} H^{4}+2(p-1)_{2}(q-1)_{2} H^{2} A_{q}+(q-1)_{4} A_{q}^{2}\right)
\end{aligned}
$$




$$
\begin{aligned}
& +4 \alpha_{4}\left((p-1)_{6} H^{6}+3(p-1)_{4}(q-1)_{2} H^{4} A_{q}+3(p-1)_{2}(q-1)_{4} H^{2} A_{q}^{2}+(q-1)_{6} A_{q}^{3}\right), \\
Z:= & 1+2\left((p+1)_{0} H^{2}+(q-3)_{4} A_{q}\right)+3 \alpha_{3}\left((p+1)_{2} H^{4}+2(p+1)_{0}(q-3)_{4} H^{2} A_{q}+(q-3)_{6} A_{q}^{2}\right) \\
& +4 \alpha_{4}\left((p+1)_{4} H^{6}+3(p+1)_{2}(q-3)_{4} H^{4} A_{q}+3(p+1)_{0}(q-3)_{6} H^{2} A_{q}^{2}+(q-3)_{8} A_{q}^{3}\right) .
\end{aligned}
$$

Eliminating $\xi$, we find a single equation:

$$
\ddot{\eta}+p H \dot{\eta}+C \eta=0,
$$

where

$$
C:=\frac{P W-R U}{P T-S Q}=\frac{A_{q}\left[(p+1)_{0} q H^{2} Y^{2}-(p-1)(q-1)_{2} A_{q} X Z\right]}{p Y\left[(p-1) H^{2} X-q A_{q} Y\right]} .
$$

Setting $\eta=\eta_{0} e^{\omega t}$, we obtain the equation for the eigenvalue $\omega$ as

$$
\omega^{2}+p H \omega+C=0 .
$$

If $\omega>0$ (or $\Re \omega>0$ ), then the perturbation is unstable. Hence we find the stability condition for the expanding universe $(H>0)$ as

(1) both eigenvalues are negative, i.e.,

$$
p^{2} H^{2}-4 C \geq 0, \quad C>0
$$

or

(2) the eigenvalues are complex conjugate numbers with negative real part (for $p H>0$ ), i.e.,

$$
p^{2} H^{2}-4 C<0 .
$$

Altogether we find the stability condition is just $C>0$.

The difference from the case only with the GB term is the definition of $X, Y$ and $Z$.

\subsection{Minkowski spacetime}

Although we are interested in a self-accelerating de Sitter spacetime, it is worth to study Minkowski spacetime, which is given by $H=0$. Eq. (3.5) or (3.8) gives

$$
\begin{aligned}
& -(q-1) A_{q}^{(\mathrm{M})}\left[1+2(q-2)_{3} A_{q}^{(\mathrm{M})}+3 \alpha_{3}(q-2)_{5}\left(A_{q}^{(\mathrm{M})}\right)^{2}\right. \\
& \left.\quad+4 \alpha_{4}(q-2)_{7}\left(A_{q}^{(\mathrm{M})}\right)^{3}\right]=0 .
\end{aligned}
$$

There are two branches: one is a trivial solution $A_{q}^{(\mathrm{M})}=0$ and the other is given by the roots of the cubic (quadratic, or linear) equation

$$
\begin{aligned}
1 & +2(q-2)_{3} A_{q}^{(\mathrm{M})}+3 \alpha_{3}(q-2)_{5}\left(A_{q}^{(\mathrm{M})}\right)^{2} \\
& +4 \alpha_{4}(q-2)_{7}\left(A_{q}^{(\mathrm{M})}\right)^{3}=0 .
\end{aligned}
$$

A trivial solution $A_{q}^{(\mathrm{M})}=0$ corresponds to $\sigma_{q}=0$, which is a torus compactification. This Minkowski spacetime with $A_{q}^{(\mathrm{M})}=0$ is always a solution.

So, in what follows, we mainly discuss the case of $A_{q}^{(\mathrm{M})} \neq 0$. We can classify the solutions as follows: 
(a) $q=2,3$ : no solution.

(b) $q=4,5$ : there exists one negative solution:

$$
A_{q}^{(\mathrm{M})}=-\frac{1}{2(q-2)_{3}} .
$$

(c) $q=6,7$ : here $\alpha_{4}$ term is absent. If $\alpha_{3} \neq 0$, there exist two solutions:

$$
A_{q}^{(\mathrm{M})}=\frac{1}{3 \alpha_{3}(q-4)_{5}}\left[-1 \pm \sqrt{1-\frac{3 \alpha_{3}(q-4)_{5}}{(q-2)_{3}}}\right]
$$

if

$$
\alpha_{3} \leq \frac{(q-2)_{3}}{3(q-4)_{5}} .
$$

For $\alpha_{3}>0$, both solutions are negative, while for $\alpha_{3}<0$, one with plus sign is negative and the other with minus sign is positive. No solution exists for

$$
\alpha_{3}>\frac{(q-2)_{3}}{3(q-4)_{5}}
$$

When $\alpha_{3}=0$, there exists one negative solution (3.22).

(d) $q \geq 8$ : three real solutions exist if

$$
\alpha_{4, \mathrm{cr}}^{(-)} \leq \alpha_{4} \leq \alpha_{4, \mathrm{cr}}^{(+)},
$$

where

$$
\alpha_{4, \mathrm{cr}}^{( \pm)}=\frac{4\left((q-2)_{3}\right)^{2}}{27(q-4)_{7}}\left[-\left(1-\frac{27 \alpha_{3}(q-4)_{5}}{8(q-2)_{3}}\right) \pm\left(1-\frac{9 \alpha_{3}(q-4)_{5}}{4(q-2)_{3}}\right)^{3 / 2}\right],
$$

with

$$
\alpha_{3} \leq \frac{4(q-2)_{3}}{9(q-4)_{5}} .
$$

For $q=8$, we show the existence range by the light-red shaded region in figure 4 .

If the condition (3.26) is not satisfied, there exists only one real solution (shown by the white region in figure 4). It is negative for $\alpha_{4}>0$ while positive for $\alpha_{4}<0$.

When $\alpha_{4}=0$, we find the same solutions as in the case (c). For $q=8$, it is also shown by the red solid (two solutions) and dashed lines (no solution) in figure 4 .

\subsection{Stability of near-Minkowski spacetime}

To analyze stability, we first consider spacetimes near Minkowski spacetime. It may be important because the realistic inflation predicts that the Hubble expansion rate $H$ must be much smaller than the Planck scale (a natural scale of vacuum expectation value of fundamental fields, which acts as a cosmological constant). 


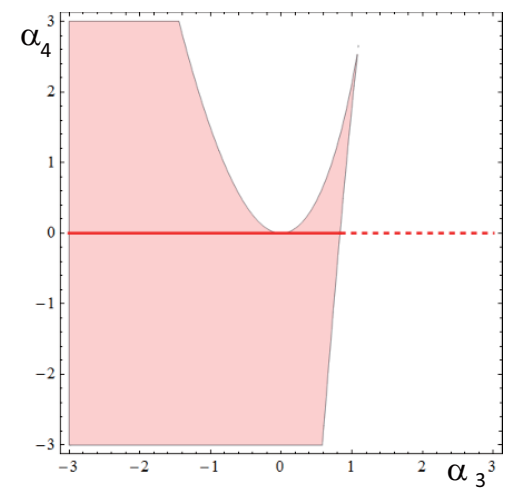

Figure 4. Three solutions exist in the light-red shaded region for $q=8$, while there exists only one solution in the white region. On the red solid and dashed lines, two solutions and no solution exist, respectively.

Near a trivial Minkowski spacetime with $A_{q}^{(\mathrm{M})}=0$, we have

$$
\begin{aligned}
A_{q} & =\frac{p}{q-1} H^{2}+O\left(H^{4}\right) \\
\Lambda & =\frac{p(p+q-1)}{2} H^{2}+O\left(H^{4}\right) .
\end{aligned}
$$

Using this approximate solution, we find the equation for $\omega(3.17)$ as

$$
\omega^{2}+p H \omega-2 p H^{2}=0
$$

which has one positive and one negative roots for $H \neq 0$. Hence the solution is always unstable.

Next we analyze another branch with $A_{q}^{(\mathrm{M})} \neq 0$. Expanding $A_{q}$ as

$$
A_{q}=A_{q}^{(\mathrm{M})}+A_{q}^{(2)} H^{2}+O\left(H^{4}\right)
$$

where $A_{q}^{(\mathrm{M})}$ is given by the solution of eq. (3.21), we find the solutions near Minkowski by (3.32) with

$$
A_{q}^{(2)}=-\frac{p\left[(p q-p-3 q+5)+3 \alpha_{3}(p q-p-3 q+9)(q-2)_{3} A_{q}^{(\mathrm{M})}+6 \alpha_{4}(p q-p-3 q+13)(q-2)_{4}\left(A_{q}^{(\mathrm{M})}\right)^{2}\right]}{(q-1)_{3}\left[1+3 \alpha_{3}(q-4)_{5} A_{q}^{(\mathrm{M})}+6 \alpha_{4}(q-4)_{7}\left(A_{q}^{(\mathrm{M})}\right)^{2}\right]} .
$$

The cosmological constant is given by

$$
\Lambda=\Lambda^{(\mathrm{M})}+\frac{p_{1}}{2} X^{(\mathrm{M})} H^{2}+O\left(H^{4}\right)
$$

where

$$
\begin{aligned}
\Lambda^{(\mathrm{M})} & \left.:=\frac{q_{1}}{2} A_{q}\left[1+(q-2)_{3} A_{q}^{(\mathrm{M})}+\alpha_{3}(q-2)_{5}\left(A_{q}^{(\mathrm{M})}\right)^{2}\right]\right] \\
X^{(\mathrm{M})} & :=X\left(H=0, A_{q}=A_{q}^{(\mathrm{M})}\right) .
\end{aligned}
$$




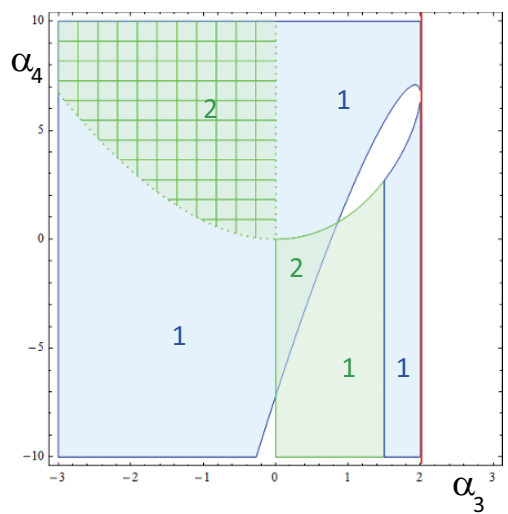

Figure 5. Stable (light-blue, light-green and meshed light-green) regions of Minkowski spacetime for $D=10(q=6)$. The numbers denote how many solutions are stable. The blue and green regions correspond to negative and positive cosmological constants, respectively, whereas the meshed and un-meshed regions to $A_{q}^{(\mathrm{M})}>0$ and $A_{q}^{(\mathrm{M})}<0$, respectively. There is no stable solution in the white region with $\alpha_{3} \leq 2$. No solution exists beyond $\alpha_{3}=2$ (the red line).

Assuming $H^{2} \ll 1$, the coefficients $C$ in (3.17) is rewritten as

$$
C=\frac{(p-1)(q-1)_{2} A_{q}^{(\mathrm{M})} X^{(\mathrm{M})} Z^{(\mathrm{M})}}{p q\left(Y^{(\mathrm{M})}\right)^{2}}+O\left(H^{2}\right),
$$

where

$$
\begin{aligned}
& Y^{(\mathrm{M})}=Y\left(H=0, A_{q}=A_{q}^{(\mathrm{M})}\right) \\
& Z^{(\mathrm{M})}=Z\left(H=0, A_{q}=A_{q}^{(\mathrm{M})}\right) .
\end{aligned}
$$

We find the eigenvalues

$$
\omega_{ \pm}=\frac{1}{2}(-p H \pm \sqrt{-4 C})+O\left(H^{2}\right) .
$$

If $C \geq 0$, the expanding de Sitter spacetime is stable.

For $q=4,5$, the stability condition amounts to

$$
\alpha_{3} \leq \frac{8(2 q-3)}{3 q_{1}}
$$

Note that $A_{q}^{(\mathrm{M})}$ is always negative just as the case only with the GB term.

For $q=6,7$, we give one example for the case of $D=10(q=6)$ in figure 5 . We show the parameter ranges according to how many stable Minkowski solutions exist by the light-blue, light-green and meshed light-green shaded regions in the $\alpha_{3}-\alpha_{4}$ plane. The numbers denote how many stable solutions exist. In the light-blue regions, $\Lambda^{(\mathrm{M})}<0$ as well as $A_{q}^{(\mathrm{M})}<0$, which is the same as the case only with the GB term. On the other hand, the light-green region corresponds to the solutions with $\Lambda^{(\mathrm{M})}>0$ as well as $A_{q}^{(\mathrm{M})}<0$, and the meshed lightgreen regions does those with $\Lambda^{(\mathrm{M})}>0$ but $A_{q}^{(\mathrm{M})}<0$, respectively. Those green regions with a positive cosmological constant appear in the minus branch of the solution (3.23). 


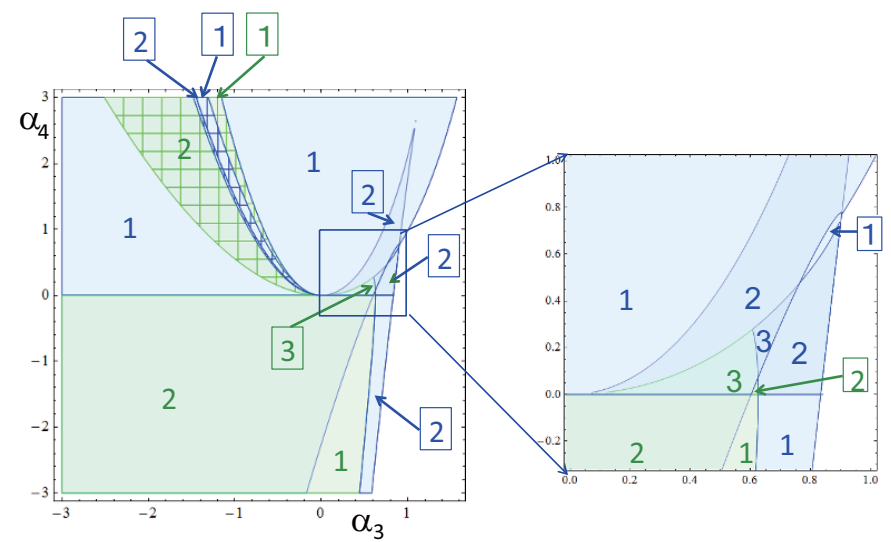

Figure 6. Stable (light-blue, meshed light-blue, light-green and meshed light-green) regions of Minkowski spacetime for $D=12(q=8)$. The numbers denote how many solutions are stable. The blue and green regions give a negative and positive cosmological constant, respectively. The meshed and un-meshed regions correspond to $A_{q}^{(\mathrm{M})}>0$ and $A_{q}^{(\mathrm{M})}<0$, respectively. There is no stable solution in the white region.

We can show that $\Lambda^{(\mathrm{M})}<0$ and $A_{q}^{(\mathrm{M})}<0$ for the plus branch of the solution (3.23), while in the minus branch,

$$
\begin{aligned}
& \Lambda^{(\mathrm{M})}<0 \text { for } \frac{(q-2)_{3}}{4(q-4)_{5}}<\alpha_{3}<\frac{(q-2)_{3}}{3(q-4)_{5}} \\
& \Lambda^{(\mathrm{M})}>0 \text { for } \alpha_{3}<\frac{(q-2)_{3}}{4(q-4)_{5}} .
\end{aligned}
$$

Hence, unlike the case only with the GB term, we obtain stable de Sitter solutions near Minkowski spacetime not only for a negative cosmological constant but also for a positive one.

We also show the parameter region of stable Minkowski solutions for $D=12(q=8)$ in figure 6. The numbers denote how many solutions are stable. The blue and green regions give a negative and positive cosmological constant, respectively. The meshed and un-meshed regions correspond to $A_{q}^{(\mathrm{M})}>0$ and $A_{q}^{(\mathrm{M})}<0$, respectively. Although the figure is complicated, the result is similar to the case of $D=10$.

In the next section, we study de Sitter solutions and their stabilities for the case with cubic and quartic Lovelock gravity terms.

\section{De Sitter spacetimes with higher-order Lovelock terms and their sta- bility}

As we discussed in sections 2.1, 2.2 and 2.3, in the theory with GB term and a cosmological constant, we find two branches: the branch (1) gives (de Sitter spacetime) $\times($ an internal space with a positive curvature), which is unstable, and the branch (2) is (de Sitter spacetime $) \times($ an internal space with a negative curvature), which is stable. In this section, including higher-order Lovelock terms, we discuss the effect of higher-order terms. Following the previous discussion about near-Minkowski spacetime, we consider three cases: (1) 
$D=8(p=3, q=4),(2) D=10(p=3, q=6)$, and (3) $D=12(p=3, q=8)$. Note that $D=10$ is predicted by superstring theory.

Solving the quadratic equation (3.8), we find the Hubble expansion parameter $H$ as

$$
H^{2}=H_{ \pm}^{2}:=\frac{-3\left[1+2(q-4)(q-1) A_{q}+3 \alpha_{3}(q-8)(q-1)_{3} A_{q}^{2}+4 \alpha_{4}(q-12)(q-1)_{5} A_{q}^{3}\right] \pm \sqrt{\mathcal{D}}}{24\left[1+3 \alpha_{3}(q-1)_{2} A q+6 \alpha_{4}(q-1)_{4} A q^{2}\right]}
$$

with

$$
\begin{aligned}
\mathcal{D}:= & 48(q-1) A_{q}\left[1+2(q-2)_{3} A_{q}+3 \alpha_{3}(q-2)_{5} A_{q}^{2}\right]\left[1+3 \alpha_{3}(q-1)_{2} A_{q}+6 \alpha_{4}(q-1)_{4} A_{q}^{2}\right] \\
& +9\left[1+2 A_{q}(q-4)(-1+q)+3 \alpha_{3} A_{q}^{2}(q-8)(q-1)_{3}+4 \alpha_{4} A_{q}^{3}(q-12)(q-1)_{5}\right]^{2} .
\end{aligned}
$$

$H_{ \pm}^{2}$ as well as $\mathcal{D}$ must be positive to find a real Hubble parameter. These conditions restrict the existence of the de Sitter solution. The cosmological constant is given in terms of $A_{q}$ by the solution (4.1) as

$$
\Lambda=\Lambda_{ \pm}:=3 H_{ \pm}^{2}\left[1+2 q_{1} A_{q}+3 \alpha_{3} q_{3} A_{q}^{2}+4 \alpha_{4} q_{5} A_{q}^{3}\right]+\frac{q_{1}}{2} A_{q}\left[1+(q-2)_{3} A_{q}+\alpha_{3}(q-2)_{5} A_{q}^{2}\right] .
$$

The coefficient $C$ in eq. (3.17) for perturbation equations is given by

$$
C=\frac{2 A_{q}\left[6 q H^{2} Y^{2}-(q-1)_{2} A_{q} X Z\right]}{3 Y\left[2 H^{2} X-q A_{q} Y\right]} .
$$

with

$$
\begin{aligned}
X:= & 1+2 q_{1} A_{q}+3 \alpha_{3} q_{3} A_{q}^{2}+4 \alpha_{4} q_{5} A_{q}^{3}, \\
Y:= & +2\left(2 H^{2}+(q-1)_{2} A_{q}\right) \\
& +3 \alpha_{3}(q-1)_{2}\left(4 H^{2}+(q-3)_{4} A_{q}\right) A_{q} \\
& +4 \alpha_{4}(q-1)_{4}\left(6 H^{2}+(q-5)_{6} A_{q}\right) A_{q}^{2}, \\
Z:= & 1+2\left(12 H^{2}+(q-3)_{4} A_{q}\right) \\
& +3 \alpha_{3}\left(24 H^{4}+24(q-3)_{4} H^{2} A_{q}+(q-3)_{6} A_{q}^{2}\right) \\
& +144 \alpha_{4}(q-3)_{4}\left(2 H^{2}+(q-5)_{6} A_{q}\right) H^{2} A_{q} .
\end{aligned}
$$

The stability condition for an expanding universe is $C \geq 0$, which is the same as the case only with the GB term. The difference is the definition of $X, Y$, and $Z$.

In the followings, we show numerical results. We analyze two limited cases: A. $\alpha_{3}=0$, and B. $\alpha_{4}=0$, and discuss more general cases in $\mathbf{C}$.

\subsection{The effect of the quartic Lovelock term with $\alpha_{4}\left(\alpha_{3}=0\right)$}

In this subsection, we discuss the effect of the quartic Lovelock term with the coupling constant $\alpha_{4}$. For $D=8$, no quartic Lovelock term appears. Then we first discuss the case of $D=10$. In figure 7 , we summarize our result on the $\alpha_{4}-A_{6}$ plane. The reason why we choose the value of $A_{6}$ to describe the solutions is because just the Hubble parameters $H_{ \pm}^{2}$ 


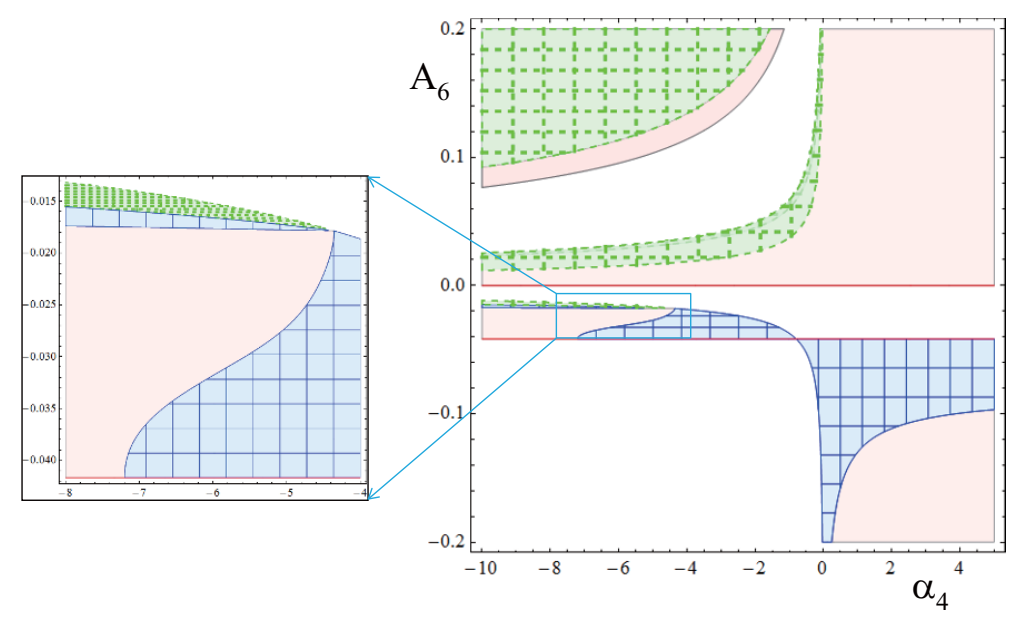

Figure 7. The de Sitter solution exists in the colored region on the $\alpha_{4}-A_{6}$ plane for $D=10$ $\left(\alpha_{3}=0\right)$. The meshed blue and meshed dotted green regions give the stable dS solutions with a negative and positive cosmological constants, respectively. The dS solution in the light-red shaded region is unstable. The red lines at $A_{6}=0$ and at $A_{6}=-\frac{1}{24}$ denote Minkowski spacetimes. The left small figure is the enlarged one of the part of the right figure.

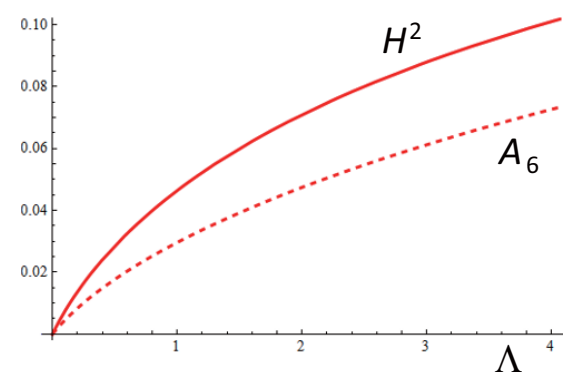

(a) branch (1)

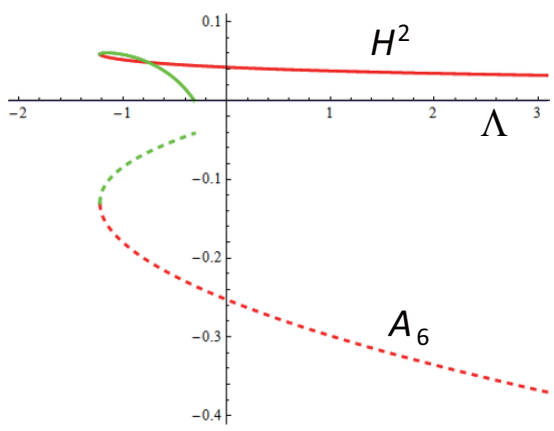

(b) branch (2)

Figure 8. The de Sitter solutions $\left(H^{2}\right.$ :Solid curves) with a static extra dimensions $\left(A_{6}\right.$ : dashed curves) in terms of a cosmological constant $\Lambda$ for two branches (branch (1) and branch (2)) in the case of $\alpha_{4}=1$. The stable solutions are denoted by the green curves, while the unstable ones are by the red ones.

and the cosmological constant $\Lambda_{ \pm}$are uniquely determined by giving the value of $A_{6}$. The de Sitter solution exists in the colored regions: the meshed blue and meshed dotted green regions give the stable dS solutions with a negative and positive cosmological constants, respectively. The $\mathrm{dS}$ solution in the light-red shaded region is unstable.

From this figure, we can classify the solutions into the following four cases (A)-(D):

(A) $\boldsymbol{\alpha}_{\mathbf{4}}>\mathbf{0}$. There exists stable de Sitter solutions with a negative cosmological constant for a finite negative range of $A_{6}$. The solutions with positive $A_{6}$ or with large negative $A_{6}$ are unstable. There exists one stable Minkowski spacetime for $A_{6}=-1 / 24$. In figure 8 , we show one example for $\alpha_{4}=1$. For the branch (2), $A_{6}$ is always negative as the case only with GB term, but a cosmological constant becomes positive for the large negative 


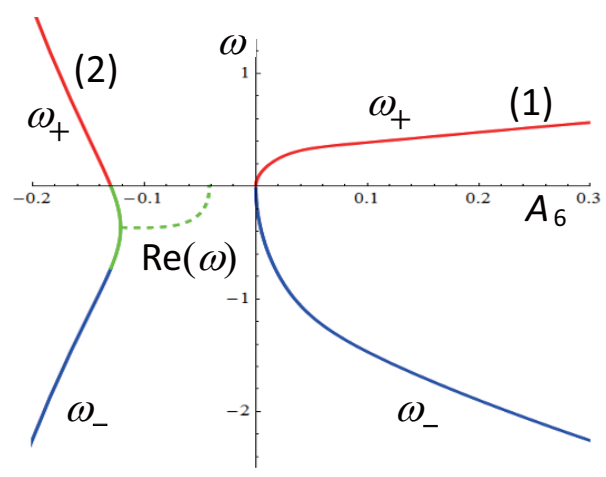

Figure 9. The eigenvalues $\omega$ in terms of $A_{6}$ for $\alpha_{4}=1$. For a given value of $A_{6}$, the green solid and dashed curves give two negative and negative real part of two complex conjugate eigenvalues, respectively, while the red and blue curves are positive and negative eigenvalues, respectively. The solutions given by the green curves in the branch (2) are stable, otherwise unstable.

value of $A_{6}$. On the other hand, for the branch (1), we find $A_{6} \geq 0$ and $\Lambda \geq 0$, which are the same as the case only with GB term.

We show the eigenvalues in figure 9 to see the stability of the solutions. The green curves give two stable modes, i.e. two negative eigenvalues or negative real part of two complex conjugate eigenvalues. These solutions are stable. On the other hand, the red and blue curves denote the unstable and stable modes, respectively. Hence such solutions are unstable. This result means that the solutions in the branch (1) are unstable and those in the branch (2) are stable for near-Minkowski spacetime. It shows the same behaviour as those in the theory only with GB term. However, the solutions in the branch (2) turn to be unstable when the curvature scale of the extra dimensions $\left(\left|A_{6}\right|\right)$ increases beyond a critical value (compare with figure 3 ).

For the other positive values of $\alpha_{4}$, we find similar results, i.e., there exists one stable branch of de Sitter solutions, for which $A_{6}$ and $\Lambda$ are always negative.

(B) $\mathbf{- 1 9 6 / 4 5}<\boldsymbol{\alpha}_{\mathbf{4}}<\mathbf{0}$. There exists de Sitter solution with a negative cosmological constant for a finite negative region of $A_{6}$. For the positive value of $A_{6}$, there are two de Sitter solutions: one is unstable and the other is stable, for which a cosmological constant is positive. Only one stable Minkowski spacetime is possible for $A_{6}=-1 / 24$.

(C) $-\mathbf{3 6} / \mathbf{5}<\boldsymbol{\alpha}_{\mathbf{4}}<\mathbf{- 1 9 6 / 4 5}$. This region is rather complicated. Changing the value of $A_{6}$, the stability and the sign of the cosmological constant changes frequently. We show one complex example with $\alpha_{4}=-6$ in figure 10. There are three branches: (1) which includes a trivial Minkowski spacetime with $A_{6}=0,(2)$ which include a stable Minkowski spacetime with $A_{6}=-1 / 24$, and (3) which newly appears and does not involve a Minkowski spacetime. The eigenvalues are shown in figure 11, from which we find the stability of the solutions.

There exists one stable de Sitter solution with a negative cosmological constant. The cosmological constant should be in a finite range of negative values. In addition, we find three stable de Sitter solutions, which belong to each branch, for large positive value of the 


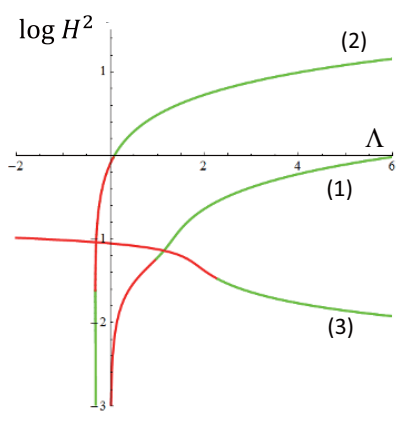

(a) $\Lambda-\log _{10} H^{2}$

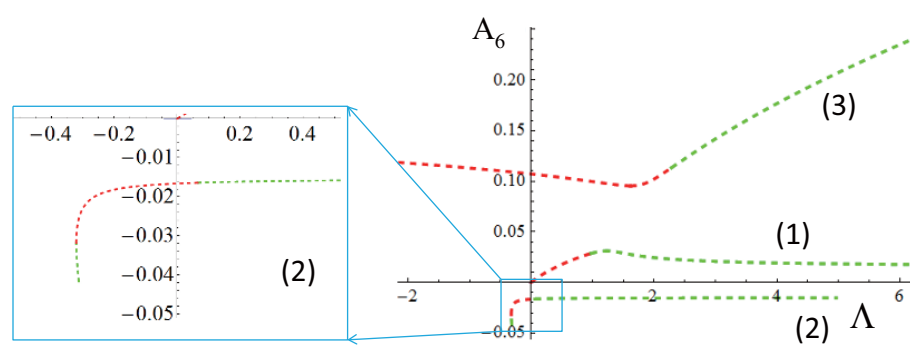

(b) $\Lambda-A_{6}$

Figure 10. The de Sitter solutions $\left(H^{2}\right)$ with a constant internal space $\left(A_{6}\right)$ in terms of a cosmological constant $\Lambda$ for $\alpha_{4}=-6$. There are three branches (the branch (1), branch (2) and branch (3), which newly appears and does not involve a Minkowski spacetime). The stable solutions are denoted by the green curves, while the unstable ones are by the red ones.

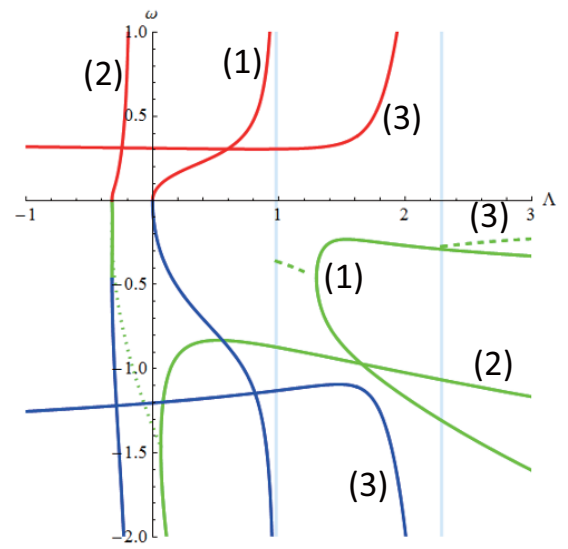

(a) The eigenvalues in three branches (1), (2) and (3)

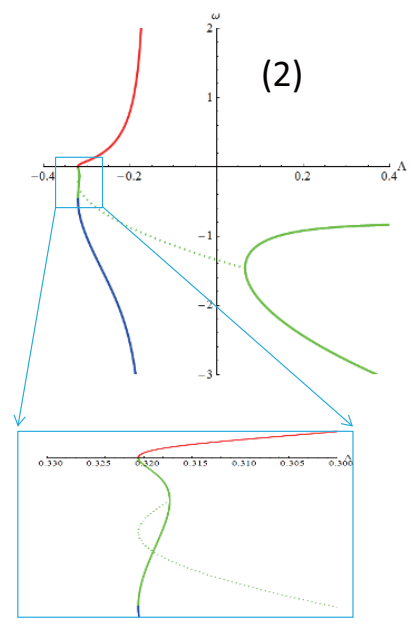

(b) The enlarged figure of the branch (2)

Figure 11. The eigenvalues in three branches (1), (2) and (3) in terms of a cosmological constant $\Lambda$ for $\alpha_{4}=-6$. The green solid and dotted curves denote two negative eigenvalues and a positive real part of two complex conjugates, respectively, which means those are stable solutions. The red and blue curves denote positive and negative eigenvalues, respectively. Those solutions are unstable.

cosmological constant. These solutions are interesting because some solutions provide us the possibility of rather small Hubble parameter in spite of large value of a cosmological constant, which may explain the discrepancy between a preferred scale of inflation (GUT scale) and the Planck scale. For a given cosmological constant with the Planck scale, it is possible that the Hubble expansion scale can be much lower.

(D) $\alpha_{\mathbf{4}}<-\mathbf{3 6} / \mathbf{5}$. The behaviour of the solutions is almost the same as the case [3], but de Sitter solution near Minkowski spacetime becomes unstable.

We also show the case of $D=12$ in figure 12 . The detail structure is very complicated, but the global feature does not change so much. The features are as follows: for $\alpha_{4}>$ 


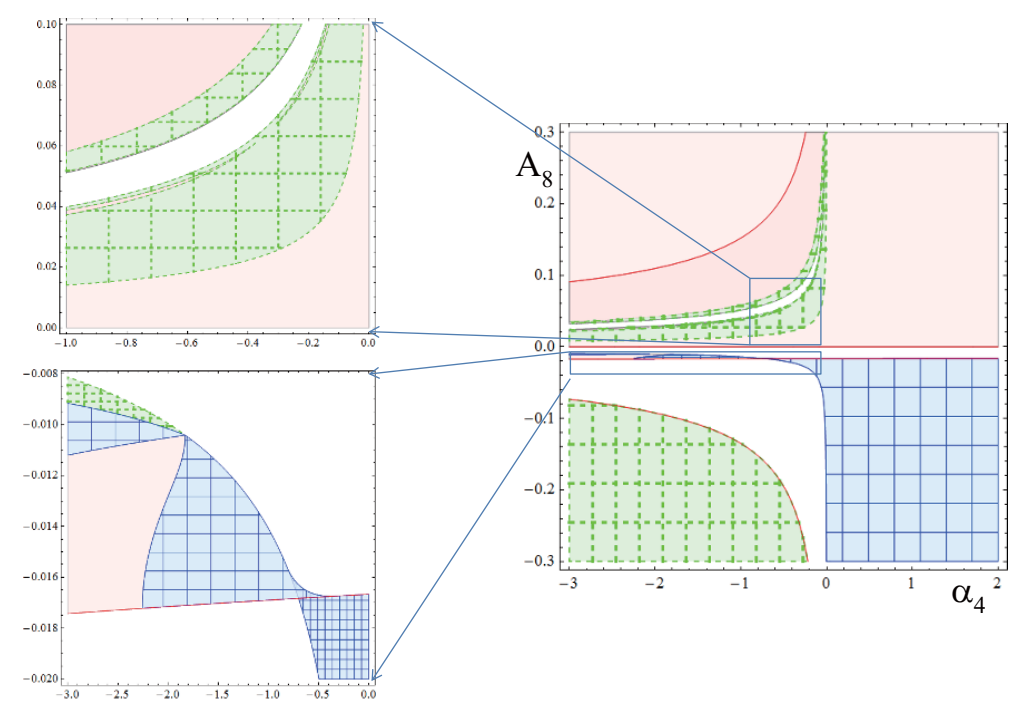

Figure 12. The de Sitter solution exists in the colored region on the $\alpha_{4}-A_{8}$ plane for $D=12$ $\left(\alpha_{3}=0\right)$. The meshed blue and meshed dotted green regions give the stable $\mathrm{dS}$ solutions with a negative and positive cosmological constants, respectively. The dS solution in the light-red shaded region is unstable. The red curves denote Minkowski spacetime. The left small figure is the enlarged one of the part of the right figure.

0, there exists a stable de Sitter solution with a negative cosmological constant for any negative $A_{8}$. If $\alpha_{4}<0$, we also find stable de Sitter solutions with negative values of $A_{8}$, but the cosmological constant must be positive.

\subsection{The effect of the cubic Lovelock term with $\alpha_{3}\left(\alpha_{4}=0\right)$}

For the case with the cubic Lovelock term $\left(\alpha_{3} \neq 0\right)$ but without the quartic term $\left(\alpha_{4}=0\right)$, we summarize our result on the $\alpha_{4}-A_{q}$ plane for $D=8,10$ and 12 in figures 13 (a)-(c), respectively.

In the case of $D=8$, for $A_{4}<0$, there exist stable de Sitter solutions (including Minkowski spacetime) with $\Lambda<0$ if $\alpha_{3}<\frac{10}{9}$. On the other hand, for $A_{4}>0$, although there are a few stable de Sitter solutions with $\Lambda>0$, most de Sitter solutions are unstable. For the cases of $D=10$ and $D=12$, apart from the small fine structures, the global features of figures 13 (b) and (c) are very similar. The de Sitter solutions with $A_{q}<0$ are mostly stable, and $\Lambda<0$ for $\alpha_{3}<0$, while $\Lambda>0$ for $\alpha_{3}>0$. On the other hand, the solutions with $A_{q}>0$ are unstable except for a few tuned solutions. Minkowski spacetime with a negative $A_{q}$ are mostly stable except for a small range of parameters (a part of the red curve next to the unstable light-red region), which is found in the enlarged figures of figures 13 (b) and (c).

From these figures, we can draw the following conclusions:

(1) There exist stable de Sitter solutions with a negative $A_{q}$ and a negative cosmological constant for $\alpha_{3}<0$.

(2) There exist stable de Sitter solutions with a negative $A_{q}$ and a positive cosmological constant for $\alpha_{3}>0$ if $D \geq 10$. 


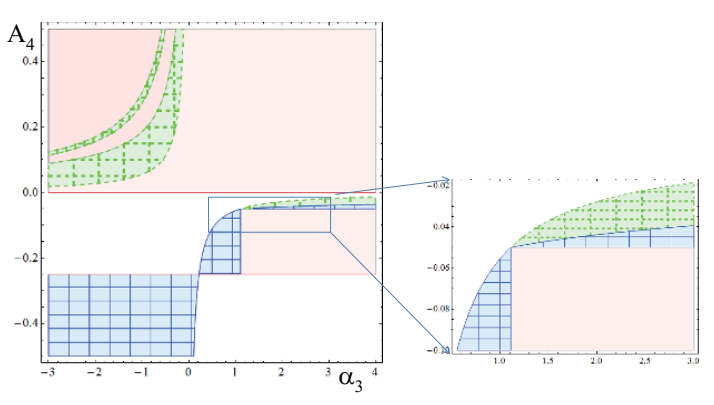

(a) $D=8$

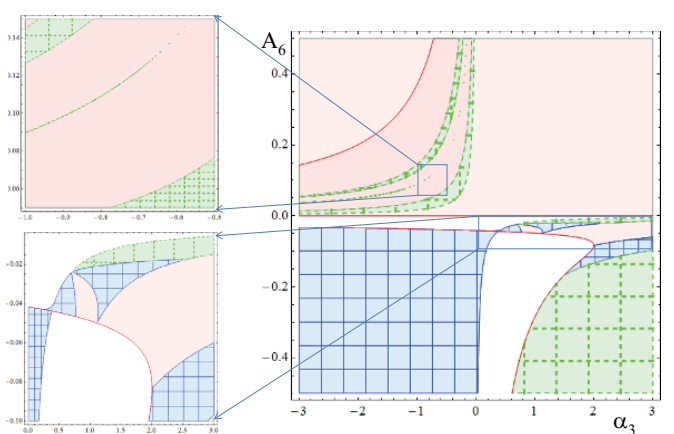

(b) $D=10$

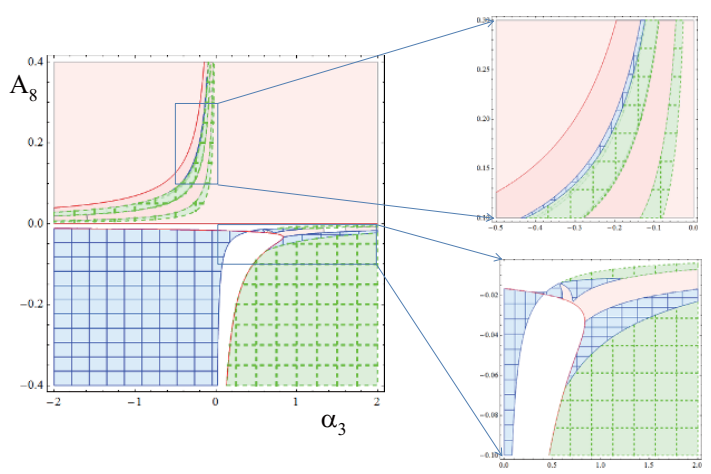

(c) $D=12$

Figure 13. The de Sitter solution exists in the colored regions on the $\alpha_{3}-A_{q}$ plane for (a) $D=8$ (b) $D=10$ and (c) $D=12$. The meshed blue and meshed dotted green regions give the stable dS solutions with a negative and positive cosmological constants, respectively. The dS solution in the light-red shaded region is unstable. The red curves denote Minkowski spacetime. The left and right small figures are the enlarged ones of the corresponding parts of the main figures.

(3) There exist a few stable de Sitter solutions with a positive $A_{q}$. Most solutions are unstable.

From figures 13, we can find the sign of $\Lambda$, but do not know the precise values. Since we are interested in the discrepancy between $H$ and $\Lambda$ in an inflationary scenario, we also show typical solutions for $D=10$ in figure 14 and 15 for some given coupling constant $\alpha_{3}$.

For the case with $\alpha_{3}=2$ in figure 14, the branches (2) and $(2)^{\prime}$ have stable de Sitter spacetimes with negative $A_{q}$. The cosmological constants can be negative, but they are continuously extended to positive values up to $+\infty$. Hence although de Sitter solution is possible for a negative cosmological constant, we also find that with a positive cosmological constant, the branch (2) may give us small Hubble parameter for a Planck scale cosmological constant, which is preferred inflation.

On the other hand, for the case with $\alpha_{3}=-2$ in figure 15, one branch (2) gives a stable de Sitter solution with negative $A_{q}$ and a negative cosmological constant, which is unbounded from below. The Hubble expansion scale can be small compared with the 


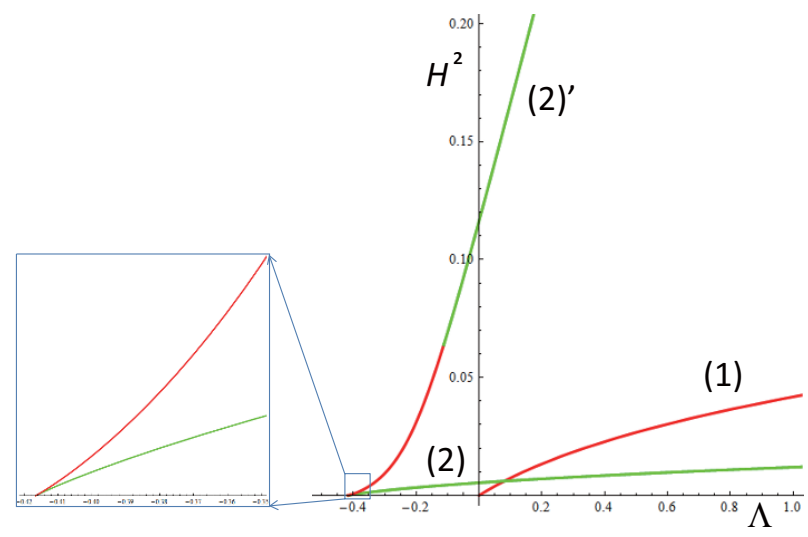

(a) $H^{2}$

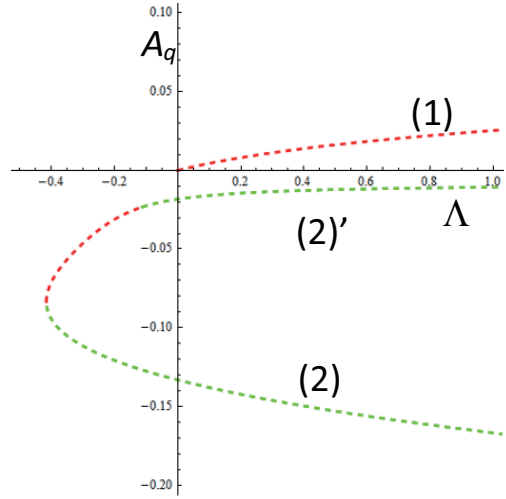

(b) $A_{q}$

Figure 14. The solution for $\alpha_{3}=2$. There are two branches of solutions (1) and (2), as shown in the figure. We also find the branch $(2)^{\prime}$ similar to the branch (2), but it includes an unstable Minkowski spacetime. The solutions denoted by the green solid and dotted curves are stable, while the red ones are unstable.

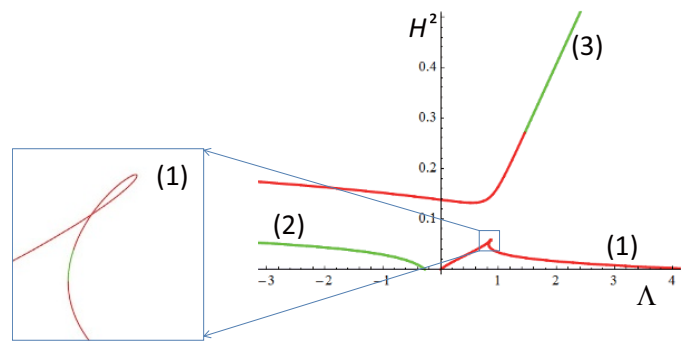

(a) $H^{2}$

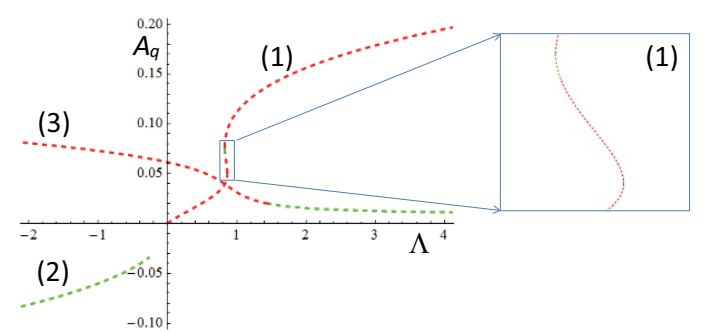

(b) $A_{q}$

Figure 15. The solution for $\alpha_{3}=-2$. There are three branches of solutions (1), (2) and (3), as shown in the figures. The branch (3) has no Minkowski spacetime. The solutions denoted by the green solid and dotted curves are stable, while the red ones are unstable.

negative cosmological constant. There also exists one new branch (3), which has a stable de Sitter solution with positive $A_{q}$ and a positive cosmological constant, which is unbounded from above. The possibility of small Hubble parameter for inflation may not be found in the branch (3), because $H$ diverges as $\Lambda \rightarrow \infty$.

To summarize, we have stable de Sitter solutions with a negative cosmological constant when $\alpha_{3}$ is negative.

\subsection{The effect of generic Lovelock terms $\left(\alpha_{3}, \alpha_{4} \neq 0\right)$}

To confirm the above results on the effects of the cubic and quartic Lovelock gravity terms, we perform calculations for the generic case with $D=10$. We show the results in figure 16 for given $\alpha_{4}$ and in figure 17 for given $\alpha_{3}$.

In figure 16, setting $\alpha_{4}=-10,-1,0,1,10$, we present the existence region of de Sitter solutions and their stabilities on the $\alpha_{3}-A_{6}$ plane. The meshed blue and meshed dotted green regions give the stable dS solutions with a negative and positive cosmological con- 


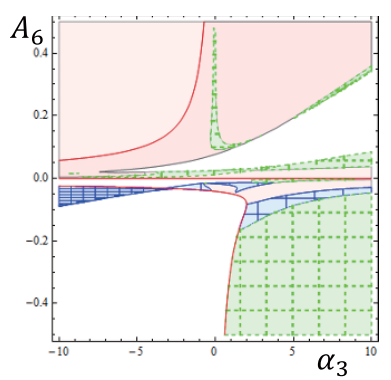

(a)

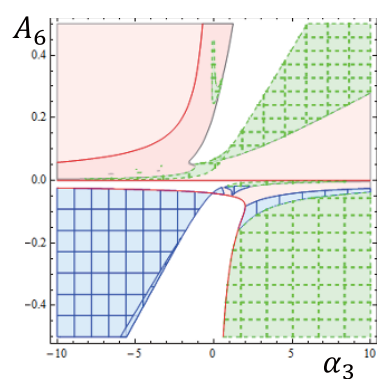

(b)

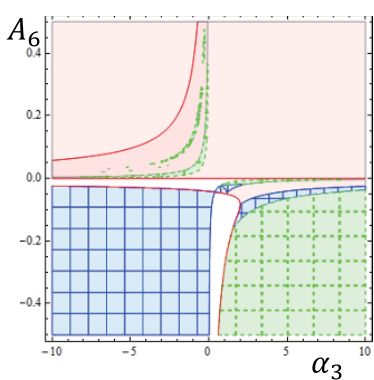

(c)

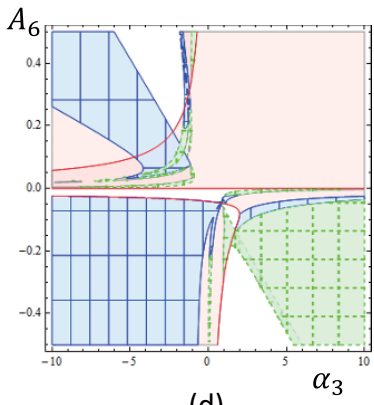

(d)

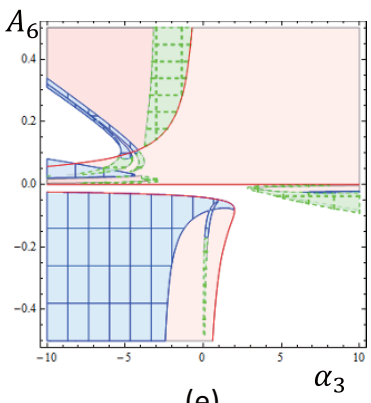

(e)

Figure 16. For given $\alpha_{4}$ [(a) $\alpha_{4}=-10$, (b) $\alpha_{4}=-1$, (c) $\alpha_{4}=0$, (d) $\alpha_{4}=1$, (e) $\left.\alpha_{4}=10\right]$, the existence of de Sitter solutions and their stabilities are shown on the $\alpha_{3}-A_{6}$ plane. The meshed blue and meshed dotted green regions give the stable dS solutions with a negative and positive cosmological constants, respectively. The dS solution in the light-red shaded region is unstable. The red curves denote Minkowski spacetime.

stants, respectively. The dS solution in the light-red shaded region is unstable. Note that the red curves denote Minkowski spacetime. The stability structure is very complicated, but we find the following overall features. We find a stable de Sitter solution with a negative cosmological constant (meshed blue region) when $\alpha_{3}<0$. In this case, $A_{6}$ is always negative, but the existence region is restricted for $\alpha_{4}<0$. On the other hand, there exists a stable de Sitter solution with a positive cosmological constant (meshed green region) when $\alpha_{3}>0$. $A_{6}$ is always negative, but the existence region is restricted for $\alpha_{4}>0$. The solutions with $A_{6}>0$ are mostly unstable.

In figure 17, setting $\alpha_{3}=-10,-1,0,1,10$, we present the similar figures on the $\alpha_{4}-A_{6}$ plane. The stability structure is again very complicated, but we also find the following global features. We find a stable de Sitter solution with a negative cosmological constant (meshed blue region) when $\alpha_{3}<0$. In this case, $A_{6}$ is mostly negative, but the restricted region appears for $\alpha_{4}<0$. On the other hand, there exists a stable de Sitter solution with a positive cosmological constant (meshed green region) when $\alpha_{3}>0 . A_{6}$ is mostly negative, but the restricted region is found for $\alpha_{4}>0$. The solutions with $A_{6}>0$ are mostly unstable.

From those figures, we can conclude that a stable de Sitter solution with a negative cosmological constant is obtained if $\alpha_{3}<0$, although the existence region of negative $A_{6}$ is constrained for $\alpha_{4}>0$. Conversely, a stable de Sitter solution with a positive cosmological constant is obtained if $\alpha_{3}>0$, although the existence region of negative $A_{6}$ is constrained for $\alpha_{4}>0$. The solutions with $A_{6}>0$ are mostly unstable. 


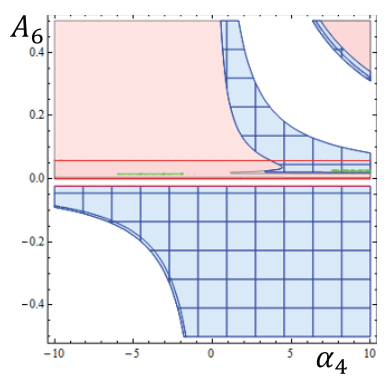

(a)

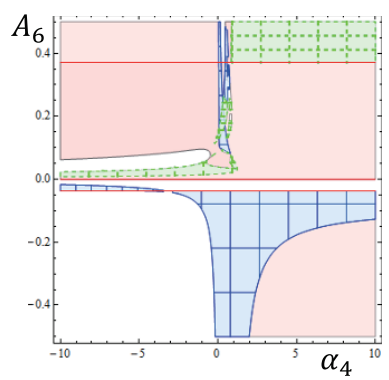

(b)

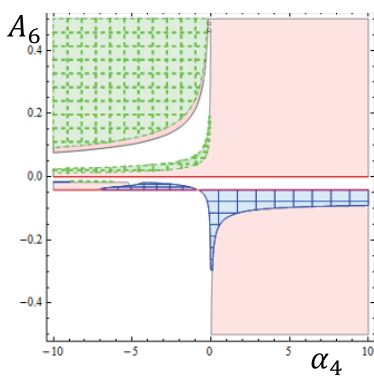

(c)

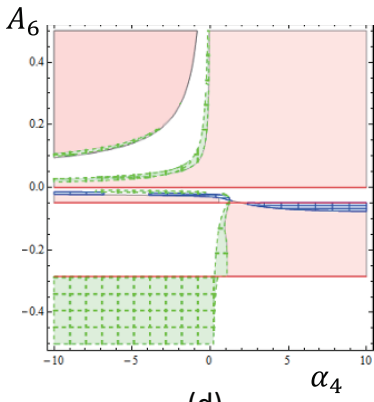

(d)

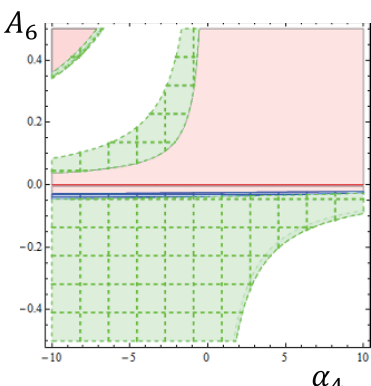

(e)

Figure 17. For given $\alpha_{3}$ [ (a) $\alpha_{3}=-10$, (b) $\alpha_{3}=-1$, (c) $\alpha_{3}=0$, (d) $\alpha_{3}=1$, (e) $\alpha_{3}=10$ ], the existence of de Sitter solutions and their stabilities are shown on the $\alpha_{4}-A_{6}$ plane. The meshed blue and meshed dotted green regions give the stable dS solutions with a negative and positive cosmological constants, respectively. The dS solution in the light-red shaded region is unstable. The red curves denote Minkowski spacetime.

Next, setting $\alpha_{3}= \pm 1$ and $\alpha_{4}= \pm 1$, we show the explicit solutions in terms of $\Lambda$ in figure 18. The green and red curves correspond to the stable and unstable solutions, respectively. From these figures, we can confirm that a stable de Sitter solution with a negative cosmological constant (branch (2) solution) exists for $\alpha_{3}=-1$, while a stable de Sitter solution with a positive cosmological constant (branch (2) and $\left.(2)^{\prime}\right)$ appears for $\alpha_{3}=$ 1. For the branch (1), a stable de Sitter spacetime appears for larger values of positive $\Lambda$.

One interesting observation is that there exist stable de Sitter solutions with large (negative or positive) cosmological constants for any coupling constants (See the branch (2) and (1) in figure 18(a), the branch (2) and (3) in figure 18(b), the branch (2) and (2)' in figures $18(\mathrm{c})$, and (d)). This may explain the discrepancy between an inflation scale and the Planck scale.

\section{Concluding remarks}

We have studied gravitational theories with a cosmological constant and the Gauss-Bonnet curvature squared term. We find that there are two branches of the de Sitter solutions: both the curvature of the internal space and the cosmological constant are (1) positive and (2) negative. By the stability analysis, we have shown that the de Sitter solution of the branch (1) is unstable, while that in the branch (2) is stable. It is remarkable that we have de Sitter solutions even for a negative cosmological constant, which are the only 

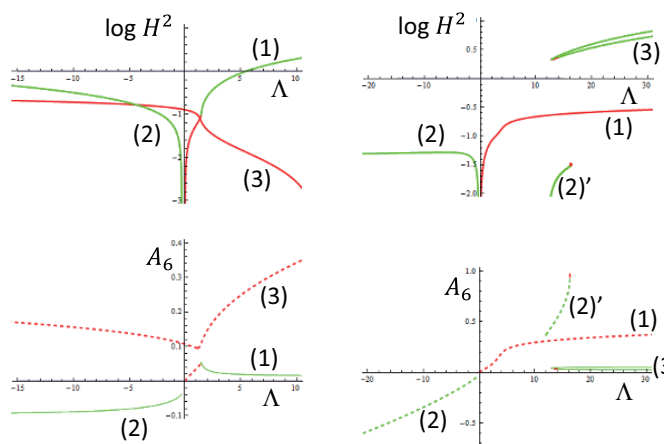

(a)

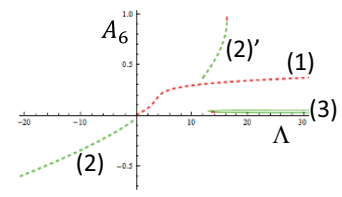

(b)
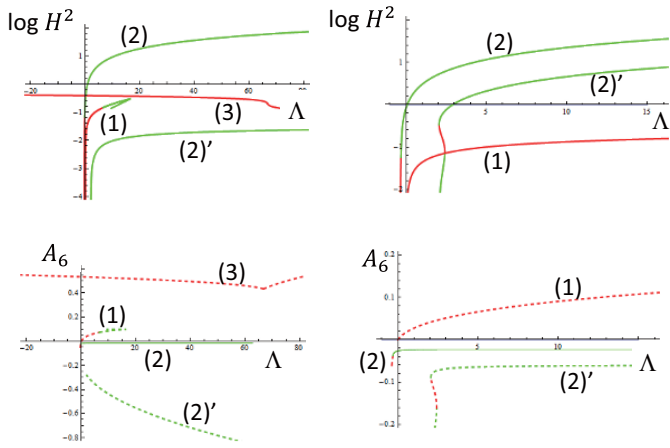

(c)

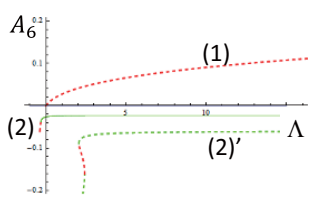

Figure 18. The de Sitter solutions for (a) $\alpha_{3}=-1, \alpha_{4}=-1$, (b) $\alpha_{3}=-1, \alpha_{4}=1$, (c) $\alpha_{3}=$ $1, \alpha_{4}=-1$, and (d) $\alpha_{3}=1, \alpha_{4}=1$. The stable solutions are shown by the green curves, while the unstable ones are by the red curves.

stable ones. We again note that we have not studied the stability of other possible moduli in extra dimensions, but the above stability is an important property for useful solutions.

We have also extended our analysis to the gravitational theories with further higherorder Lovelock curvature terms. Although the existence and the stability of the de Sitter solutions are very complicated and highly depend on the coupling constants $\alpha_{3}$ and $\alpha_{4}$, there exist stable de Sitter solutions similar to the branch (2) for $\alpha_{3}<0$. We also find stable de Sitter solutions with positive cosmological constants if $\alpha_{3}>0$. For most stable de Sitter solutions, the Hubble scale can be much smaller than the scale of a cosmological constant, which may explain a discrepancy between an inflation energy scale and the Planck scale.

Although the existence of a stable de Sitter spacetime with a negative cosmological constant is interesting, it is important to find a realistic cosmological model for the early universe, in which de Sitter exponential expansion must end at some stage. It means that de Sitter solution should be a marginally unstable state instead of an absolute stable state. After more than 60 e-foldings, inflation must end and the universe must be reheated, finding a big bang initial state. Hence we have to find a graceful exist in the present model. Only after such a mechanism is found, we can discuss density perturbations and observational consequences.

There is another point to be discussed. We have shown that there are two (or more) branches of the de Sitter solutions. One branch (the branch (1)) is connected to the solutions of general relativity (GR) in the limit of $\alpha_{2} \rightarrow 0$. We call it GR-branch. The other branches (the branch (2), (2)' and (3)) are called non-GR branches, because there is no GR limit for any values of the coupling constants [73-75]. Since the present universe is well described by GR, it may be plausible that the realistic cosmological solutions belong to the GR branch. This may mean either that we should find an interesting solution in the branch (1) [for example, there exists a stable de Sitter spacetime with $\Lambda<0$ and $A_{q}<0$ in the branch (1) for $\alpha_{3}=0, \alpha_{4}=-6$ in figure 10], or that we should construct a realistic cosmological model including a low-energy scale universe in the other branches. These are under investigation. 
The present model may be too simple from the viewpoint of a unified theory of fundamental interactions. It may be desirable to analyze more realistic models based on supergravity or superstring theory including a dilaton field.

\section{Acknowledgments}

We would like to thank Nathalie Deruelle and Hideki Maeda for useful comments. KM would like to acknowledge hospitality of Department of Physics, University of Auckland, where this work was completed. This work was supported in part by Grants-in-Aid from the Scientific Research Fund of the Japan Society for the Promotion of Science (C) Nos. 24540290 and 25400276 , and (A) No. 22244030.

Open Access. This article is distributed under the terms of the Creative Commons Attribution License (CC-BY 4.0), which permits any use, distribution and reproduction in any medium, provided the original author(s) and source are credited.

\section{References}

[1] A.A. Starobinsky, A new type of isotropic cosmological models without singularity, Phys. Lett. B 91 (1980) 99 [INSPIRE].

[2] K. Sato, First order phase transition of a vacuum and expansion of the universe, Mon. Not. Roy. Astron. Soc. 195 (1981) 467 [INSPIRE].

[3] A.H. Guth, The inflationary universe: a possible solution to the horizon and flatness problems, Phys. Rev. D 23 (1981) 347 [INSPIRE].

[4] A. Albrecht and P.J. Steinhardt, Cosmology for Grand Unified Theories with radiatively induced symmetry breaking, Phys. Rev. Lett. 48 (1982) 1220 [InSPIRE].

[5] A.D. Linde, A new inflationary universe scenario: a possible solution of the horizon, flatness, homogeneity, isotropy and primordial monopole problems, Phys. Lett. B 108 (1982) 389 [INSPIRE].

[6] A.D. Linde, Chaotic inflation, Phys. Lett. B 129 (1983) 177 [INSPIRE].

[7] Planck collaboration, P.A.R. Ade et al., Planck 2013 results. XXII. Constraints on inflation, arXiv: 1303.5082 [INSPIRE].

[8] WMAP collaboration, D.N. Spergel et al., First year Wilkinson Microwave Anisotropy Probe (WMAP) observations: determination of cosmological parameters, Astrophys. J. Suppl. 148 (2003) 175 [astro-ph/0302209] [INSPIRE].

[9] WMAP collaboration, D.N. Spergel et al., Wilkinson Microwave Anisotropy Probe (WMAP) three year results: implications for cosmology, Astrophys. J. Suppl. 170 (2007) 377 [astro-ph/0603449] [INSPIRE].

[10] WMAP collaboration, H.V. Peiris et al., First year Wilkinson Microwave Anisotropy Probe (WMAP) observations: implications for inflation, Astrophys. J. Suppl. 148 (2003) 213 [astro-ph/0302225] [INSPIRE].

[11] Planck collaboration, P.A.R. Ade et al., Planck 2013 results. I. Overview of products and scientific results, arXiv:1303.5062 [INSPIRE]. 
[12] Planck collaboration, P.A.R. Ade et al., Planck 2013 results. XVI. Cosmological parameters, arXiv:1303.5076 [INSPIRE].

[13] Planck collaboration, P.A.R. Ade et al., Planck 2013 results. XXII. Constraints on inflation, arXiv: 1303.5082 [INSPIRE].

[14] BICEP2 collaboration, P.A.R. Ade et al., BICEP2 I: detection of B-mode polarization at degree angular scales, arXiv:1403.3985 [INSPIRE].

[15] G.W. Gibbons, Aspects of supergravity theories, in Proceedings of the GIFT seminar on theoretical physics, San Feliu de Guixols Spain June 4-11 1984, F. Del Aguila et al. eds., World Scientific, Singapore (1984), pg. 123 [INSPIRE].

[16] J.M. Maldacena and C. Núñez, Supergravity description of field theories on curved manifolds and a no go theorem, Int. J. Mod. Phys. A 16 (2001) 822 [hep-th/0007018] [INSPIRE].

[17] G.R. Dvali and S.-H. Henry Tye, Brane inflation, Phys. Lett. B 450 (1999) 72 [hep-ph/9812483] [INSPIRE].

[18] S.B. Giddings, S. Kachru and J. Polchinski, Hierarchies from fluxes in string compactifications, Phys. Rev. D 66 (2002) 106006 [hep-th/0105097] [INSPIRE].

[19] S. Kachru, R. Kallosh, A.D. Linde and S.P. Trivedi, De Sitter vacua in string theory, Phys. Rev. D 68 (2003) 046005 [hep-th/0301240] [INSPIRE].

[20] S. Kachru et al., Towards inflation in string theory, JCAP 10 (2003) 013 [hep-th/0308055] [INSPIRE].

[21] S.-H. Henry Tye, Brane inflation: string theory viewed from the cosmos, Lect. Notes Phys. 737 (2008) 949 [hep-th/0610221] [INSPIRE].

[22] C.-M. Chen, D.V. Gal'tsov and M. Gutperle, $S$ brane solutions in supergravity theories, Phys. Rev. D 66 (2002) 024043 [hep-th/0204071] [InSPIRE].

[23] N. Ohta, Intersection rules for S-branes, Phys. Lett. B 558 (2003) 213 [hep-th/0301095] [INSPIRE].

[24] P.K. Townsend and M.N.R. Wohlfarth, Accelerating cosmologies from compactification, Phys. Rev. Lett. 91 (2003) 061302 [hep-th/0303097] [INSPIRE].

[25] N. Ohta, Accelerating cosmologies from S-branes, Phys. Rev. Lett. 91 (2003) 061303 [hep-th/0303238] [INSPIRE].

[26] N. Ohta, A study of accelerating cosmologies from superstring/M theories, Prog. Theor. Phys. 110 (2003) 269 [hep-th/0304172] [INSPIRE].

[27] M.N.R. Wohlfarth, Accelerating cosmologies and a phase transition in M-theory, Phys. Lett. B 563 (2003) 1 [hep-th/0304089] [INSPIRE].

[28] D.J. Gross and J.H. Sloan, The quartic effective action for the heterotic string, Nucl. Phys. B 291 (1987) 41 [INSPIRE].

[29] M. de Roo, H. Suelmann and A. Wiedemann, The supersymmetric effective action of the heterotic string in ten-dimensions, Nucl. Phys. B 405 (1993) 326 [hep-th/9210099] [INSPIRE].

[30] A.A. Tseytlin, Heterotic type-I superstring duality and low-energy effective actions, Nucl. Phys. B 467 (1996) 383 [hep-th/9512081] [INSPIRE]. 
[31] K. Peeters, P. Vanhove and A. Westerberg, Supersymmetric higher derivative actions in ten-dimensions and eleven-dimensions, the associated superalgebras and their formulation in superspace, Class. Quant. Grav. 18 (2001) 843 [hep-th/0010167] [INSPIRE].

[32] K. Bamba, Z.-K. Guo and N. Ohta, Accelerating cosmologies in the Einstein-Gauss-Bonnet theory with dilaton, Prog. Theor. Phys. 118 (2007) 879 [arXiv:0707.4334] [InSPIRE].

[33] K.-I. Maeda, N. Ohta and R. Wakebe, Accelerating universes in string theory via field redefinition, Eur. Phys. J. C 72 (2012) 1949 [arXiv:1111.3251] [INSPIRE].

[34] S.R. Green, E.J. Martinec, C. Quigley and S. Sethi, Constraints on string cosmology, Class. Quant. Grav. 29 (2012) 075006 [arXiv:1110.0545] [InSPIRE].

[35] A.A. Starobinsky, A new type of isotropic cosmological models without singularity, Phys. Lett. B 91 (1980) 99 [INSPIRE].

[36] H. Ishihara, Cosmological solutions of the extended Einstein gravity with the Gauss-Bonnet term, Phys. Lett. B 179 (1986) 217 [INSPIRE].

[37] K.-I. Maeda, Cosmological solutions with Calabi-Yau compactification, Phys. Lett. B 166 (1986) 59 [INSPIRE].

[38] B.C. Paul and S. Mukherjee, Higher dimensional cosmology with Gauss-Bonnet terms and the cosmological constant problem, Phys. Rev. D 42 (1990) 2595 [InSPIRE].

[39] M. Gasperini and M. Giovannini, Inflation in scalar-tensor gravity with quadratic Gauss-Bonnet selfinteractions, Phys. Lett. B 287 (1992) 56 [InSPIRE].

[40] K.-I. Maeda, Inflation as a transient attractor in $R^{2}$ cosmology, Phys. Rev. D 37 (1988) 858 [INSPIRE].

[41] K.-I. Maeda, J.A. Stein-Schabes and T. Futamase, Inflation in a renormalizable cosmological model and the cosmic no hair conjecture, Phys. Rev. D 39 (1989) 2848 [INSPIRE].

[42] K.-I. Maeda, Towards the Einstein-Hilbert action via conformal transformation, Phys. Rev. D 39 (1989) 3159 [INSPIRE].

[43] S. Kachru et al., Towards inflation in string theory, JCAP 10 (2003) 013 [hep-th/0308055] [INSPIRE].

[44] K.-I. Maeda and N. Ohta, Inflation from M-theory with fourth-order corrections and large extra dimensions, Phys. Lett. B 597 (2004) 400 [hep-th/0405205] [INSPIRE].

[45] K.-I. Maeda and N. Ohta, Inflation from superstring/M-theory compactification with higher order corrections. I, Phys. Rev. D 71 (2005) 063520 [hep-th/0411093] [INSPIRE].

[46] K. Akune, K.-I. Maeda and N. Ohta, Inflation from superstring/M-theory compactification with higher order corrections. II. Case of quartic Weyl terms, Phys. Rev. D 73 (2006) 103506 [hep-th/0602242] [INSPIRE].

[47] M.H. Dehghani, Accelerated expansion of the universe in Gauss-Bonnet gravity, Phys. Rev. D 70 (2004) 064009 [hep-th/0404118] [INSPIRE].

[48] S. Nojiri, S.D. Odintsov and M. Sasaki, Gauss-Bonnet dark energy, Phys. Rev. D 71 (2005) 123509 [hep-th/0504052] [INSPIRE].

[49] G. Calcagni, S. Tsujikawa and M. Sami, Dark energy and cosmological solutions in second-order string gravity, Class. Quant. Grav. 22 (2005) 3977 [hep-th/0505193] [INSPIRE]. 
[50] S. Nojiri, S.D. Odintsov and M. Sami, Dark energy cosmology from higher-order, string-inspired gravity and its reconstruction, Phys. Rev. D 74 (2006) 046004 [hep-th/0605039] [INSPIRE].

[51] S. Tsujikawa, Cosmologies from higher-order string corrections, Annalen Phys. 15 (2006) 302 [hep-th/0606040] [INSPIRE].

[52] T. Koivisto and D.F. Mota, Cosmology and astrophysical constraints of Gauss-Bonnet dark energy, Phys. Lett. B 644 (2007) 104 [astro-ph/0606078] [INSPIRE].

[53] T. Koivisto and D.F. Mota, Gauss-Bonnet quintessence: background evolution, large scale structure and cosmological constraints, Phys. Rev. D 75 (2007) 023518 [hep-th/0609155] [INSPIRE].

[54] K. Andrew, B. Bolen and C.A. Middleton, Effects of dynamical compactification on D-dimensional Gauss-Bonnet FRW cosmology, hep-th/0608127 [INSPIRE].

[55] S. Tsujikawa and M. Sami, String-inspired cosmology: late time transition from scaling matter era to dark energy universe caused by a Gauss-Bonnet coupling, JCAP 01 (2007) 006 [hep-th/0608178] [INSPIRE].

[56] S. Nojiri and S.D. Odintsov, Modified gravity and its reconstruction from the universe expansion history, J. Phys. Conf. Ser. 66 (2007) 012005 [hep-th/0611071] [INSPIRE].

[57] G. Cognola, E. Elizalde, S. Nojiri, S. Odintsov and S. Zerbini, String-inspired Gauss-Bonnet gravity reconstructed from the universe expansion history and yielding the transition from matter dominance to dark energy, Phys. Rev. D 75 (2007) 086002 [hep-th/0611198] [INSPIRE].

[58] E. Elizalde, A.N. Makarenko, V.V. Obukhov, K.E. Osetrin and A.E. Filippov, Stationary vs. singular points in an accelerating FRW cosmology derived from six-dimensional Einstein-Gauss-Bonnet gravity, Phys. Lett. B 644 (2007) 1 [hep-th/0611213] [INSPIRE].

[59] B.M. Leith and I.P. Neupane, Gauss-Bonnet cosmologies: crossing the phantom divide and the transition from matter dominance to dark energy, JCAP 05 (2007) 019

[hep-th/0702002] [INSPIRE].

[60] L. Amendola, C. Charmousis and S.C. Davis, Solar system constraints on Gauss-Bonnet mediated dark energy, JCAP 10 (2007) 004 [arXiv:0704.0175] [INSPIRE].

[61] A. Sheykhi, B. Wang and N. Riazi, String inspired explanation for the super-acceleration of our universe, Phys. Rev. D 75 (2007) 123513 [arXiv:0704.0666] [INSPIRE].

[62] S. Nojiri, S.D. Odintsov and P.V. Tretyakov, Dark energy from modified F(R)-scalar-Gauss-Bonnet gravity, Phys. Lett. B 651 (2007) 224 [arXiv:0704.2520] [INSPIRE].

[63] E. Elizalde et al., Dark energy generated from a (super)string effective action with higher order curvature corrections and a dynamical dilaton, Eur. Phys. J. C 53 (2008) 447 [arXiv: 0705.1211] [INSPIRE].

[64] F. Canfora, A. Giacomini and S. Willison, Some exact solutions with torsion in $5 D$ Einstein-Gauss-Bonnet gravity, Phys. Rev. D 76 (2007) 044021 [arXiv:0706. 2891] [INSPIRE].

[65] Z.-K. Guo, N. Ohta and S. Tsujikawa, Realizing scale-invariant density perturbations in low-energy effective string theory, Phys. Rev. D 75 (2007) 023520 [hep-th/0610336] [INSPIRE]. 
[66] F. Canfora, A. Giacomini and S.A. Pavluchenko, Dynamical compactification in Einstein-Gauss-Bonnet gravity from geometric frustration, Phys. Rev. D 88 (2013) 064044 [arXiv: 1308.1896] [INSPIRE].

[67] J. Polchinski, String theory, Cambridge Univ. Press, Cambridge U.K. (1998).

[68] L. Álvarez-Gaumé, P.H. Ginsparg, G.W. Moore and C. Vafa, An $O(16) \times O(16)$ heterotic string, Phys. Lett. B 171 (1986) 155 [INSPIRE].

[69] V.F. Cardone, R.P. Cardenas and Y.L. Nodal, Halting eternal acceleration with an effective negative cosmological constant, Class. Quant. Grav. 25 (2008) 135010 [arXiv:0805.1267] [INSPIRE].

[70] R. Cardenas, T. Gonzalez, Y. Leiva, O. Martin and I. Quiros, A model of the universe including dark energy accounted for by both a quintessence field and a (negative) cosmological constant, Phys. Rev. D 67 (2003) 083501 [astro-ph/0206315] [INSPIRE].

[71] J.B. Hartle, S.W. Hawking and T. Hertog, Accelerated expansion from negative $\Lambda$, arXiv: 1205.3807 [INSPIRE].

[72] N. Deruelle and L. Farina-Busto, The Lovelock gravitational field equations in cosmology, Phys. Rev. D 41 (1990) 3696 [inSPIRE].

[73] H. Maeda, Universal slow fall-off to the unique AdS infinity in Einstein-Gauss-Bonnet gravity, Phys. Rev. D 78 (2008) 041503 [arXiv:0805.4025] [InSPIRE].

[74] H. Maeda and M. Nozawa, Generalized Misner-Sharp quasi-local mass in Einstein-Gauss-Bonnet gravity, Phys. Rev. D 77 (2008) 064031 [arXiv:0709.1199] [INSPIRE].

[75] M. Nozawa and H. Maeda, Dynamical black holes with symmetry in Einstein-Gauss-Bonnet gravity, Class. Quant. Grav. 25 (2008) 055009 [arXiv:0710.2709] [INSPIRE]. 